\title{
Unified triminimal parametrizations of quark and lepton mixing matrices
}

\author{
Xiao-Gang He, ${ }^{1,2,3}$ Shi-Wen $\mathrm{Li}^{1}{ }^{1}$ and Bo-Qiang $\mathrm{Ma}^{1,3, *}$ \\ ${ }^{1}$ School of Physics and State Key Laboratory of Nuclear Physics and Technology, Peking University, Beijing 100871 \\ ${ }^{2}$ Department of Physics and Center for Theoretical Sciences, National Taiwan University, Taipei 10617 \\ ${ }^{3}$ Center for High Energy Physics, Peking University, Beijing 100871
}

\begin{abstract}
We present a detailed study on triminimal parametrizations of quark and lepton mixing matrices with different basis matrices. We start with a general discussion on the triminimal expansion of the mixing matrix and on possible unified quark and lepton parametrization using quark-lepton complementarity (QLC). We then consider several interesting basis matrices and compare the triminimal parametrizations with the Wolfenstein-like parametrizations. The usual Wolfenstein parametrization for quark mixing is a triminimal expansion around the unit matrix as the basis. The corresponding QLC lepton mixing matrix is a triminimal expansion around the bimaximal basis. Current neutrino oscillation data show that the lepton mixing matrix is very well represented by the tri-bimaximal mixing. It is natural to take it as an expanding basis. The corresponding zeroth order basis for quark mixing in this case makes the triminimal expansion converge much faster than the usual Wolfenstein parametrization. The triminimal expansion based on tri-bimaximal mixing can be converted to the Wolfenstein-like parametrizations discussed in the literature. We thus have a unified description between different kinds of parametrizations for quark and lepton sectors: the standard parametrizations, the Wolfenstein-like parametrizations, and the triminimal parametrizations.
\end{abstract}

PACS numbers: 12.15.Ff, 14.60.-z, 14.60.Pq, 14.65.-q, 14.60.Lm

\section{INTRODUCTION}

Mixing between different generations of fermions in weak interaction is one of the most interesting issues in particle physics. It is characterized by an unitary matrix in the charged current interaction of W-boson in the mass eigen-state of fermions. Quark mixing is described by the Cabibbo [1]-Kobayashi-Maskawa 2] (CKM) matrix $V_{\mathrm{CKM}}$, and lepton mixing is described by the Pontecorvo [3]-Maki-Nakawaga-Sakata [4] (PMNS) matrix $U_{\mathrm{PMNS}}$ with

$$
L=-\frac{g}{\sqrt{2}} \bar{U}_{L} \gamma^{\mu} V_{\mathrm{CKM}} D_{L} W_{\mu}^{+}-\frac{g}{\sqrt{2}} \bar{E}_{L} \gamma^{\mu} U_{\mathrm{PMNS}} N_{L} W_{\mu}^{-}+H . C .,
$$

where $U_{L}=\left(u_{L}, c_{L}, t_{L}, \ldots\right)^{T}, D_{L}=\left(d_{L}, s_{L}, b_{L}, \ldots\right)^{T}, E_{L}=\left(e_{L}, \mu_{L}, \tau_{L}, \ldots\right)^{T}$, and $N_{L}=\left(\nu_{1}, \nu_{2}, \nu_{3}, \ldots\right)^{T}$ are the lefthanded fermion generations. For n-generations, $V=V_{\mathrm{CKM}}$ or $U_{\mathrm{PMNS}}$ is an $n \times n$ unitary matrix.

A commonly used form of mixing matrix for three generations of fermions is given by [5, 6 ],

$$
V=\left(\begin{array}{ccc}
c_{12} c_{13} & s_{12} c_{13} & s_{13} e^{-i \delta} \\
-s_{12} c_{23}-c_{12} s_{23} s_{13} e^{i \delta} & c_{12} c_{23}-s_{12} s_{23} s_{13} e^{i \delta} & s_{23} c_{13} \\
s_{12} s_{23}-c_{12} c_{23} s_{13} e^{i \delta} & -c_{12} s_{23}-s_{12} c_{23} s_{13} e^{i \delta} & c_{23} c_{13}
\end{array}\right),
$$

where $s_{i j}=\sin \theta_{i j}$ and $c_{i j}=\cos \theta_{i j}$ are the mixing angles and $\delta$ is the $\mathrm{CP}$ violating phase. If neutrinos are of Majorana type, for the PMNS matrix one should include an additional diagonal matrix with two Majorana phases $\operatorname{diag}\left(e^{i \alpha_{1} / 2}, e^{i \alpha_{2} / 2}, 1\right)$ multiplied to the matrix from right in the above. The two $\mathrm{CP}$ violating Majorana phases do not affect neutrino oscillations, and we ignore these phases in our discussions. To distinguish different $\mathrm{CP}$ violating phases, the phase $\delta$ is sometimes called Dirac CP violating phase.

The above unitary matrix $V$ can be expressed as

$$
V=R_{23}\left(\theta_{23}\right) U_{\delta}^{\dagger} R_{13}\left(\theta_{13}\right) U_{\delta} R_{12}\left(\theta_{12}\right),
$$

with

$$
R_{23}=\left(\begin{array}{ccc}
1 & 0 & 0 \\
0 & c_{23} & s_{23} \\
0 & -s_{23} & c_{23}
\end{array}\right), \quad R_{13}=\left(\begin{array}{ccc}
c_{13} & 0 & s_{13} \\
0 & 1 & 0 \\
-s_{13} & 0 & c_{13}
\end{array}\right), \quad R_{12}=\left(\begin{array}{ccc}
c_{12} & s_{12} & 0 \\
-s_{12} & c_{12} & 0 \\
0 & 0 & 1
\end{array}\right)
$$

*Electronic address: mabq@phy.pku.edu.cn 
and $U_{\delta}=\operatorname{diag}\left(e^{i \delta / 2}, 1, e^{-i \delta / 2}\right)$. This way of parameterizing the mixing provides a clear mathematic description of the mixing matrix with three angles describing rotations in generation space and a phase describing $\mathrm{CP}$ violation. In our later discussions, we will indicate the mixing angles with superscriptions $Q$ and $L$ for quark and lepton sectors respectively when specification is needed.

There are a lot of experimental data on the mixing patterns in both the quark and lepton sectors. For quark mixing, the ranges of the magnitudes of the CKM matrix elements have been very well determined with [6]

$$
\left(\begin{array}{ccc}
0.97419 \pm 0.00022 & 0.2257 \pm 0.0010 & 0.00359 \pm 0.00016 \\
0.2256 \pm 0.0010 & 0.97334 \pm 0.00023 & 0.0415_{-0.0011}^{+0.0010} \\
0.00874_{-0.00037}^{+0.00026} & 0.0407 \pm 0.0010 & 0.999133_{-0.000043}^{+0.000044}
\end{array}\right)
$$

From the above, we obtain the ranges for mixing angles $\theta_{i j}^{Q}$,

$$
\theta_{12}^{Q}=0.2277 \pm 0.0010, \quad \theta_{23}^{Q}=0.0415_{-0.0011}^{+0.0010}, \quad \theta_{13}^{Q}=0.00359 \pm 0.00016
$$

The CP violating phase has also been determined with $\delta^{Q} \simeq \gamma^{0}=(66.7 \pm 6.4)^{\circ}[7]$.

Considerable experimental data on lepton mixing have also been accumulated. The recent global, $1 \sigma(3 \sigma)$, fit from neutrino oscillation data gives [8],

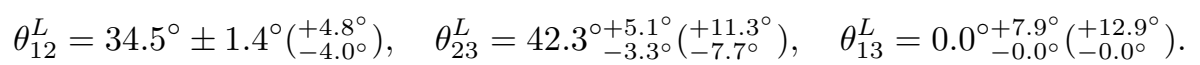

At present there is no experimental data on the CP violating Dirac phase $\delta^{L}$ and Majorana phases $\alpha_{i}$.

When studying mixing, it is interesting to parameterize $V$ according to the hierarchical structure of the mixing to reveal more physical information about the underlying theory. The Wolfenstein parametrization for quarks is a famous example of this type, where $V$ is parameterized as [9]

$$
V_{C K M}=\left(\begin{array}{ccc}
1-\frac{1}{2} \lambda^{2} & \lambda & A \lambda^{3}(\rho-i \eta) \\
-\lambda & 1-\frac{1}{2} \lambda^{2} & A \lambda^{2} \\
A \lambda^{3}(1-\rho-i \eta) & -A \lambda^{2} & 1
\end{array}\right)+\mathcal{O}\left(\lambda^{4}\right)
$$

with $\lambda=0.2257_{-0.0010}^{+0.0009}, A=0.814_{-0.022}^{+0.021}, \rho\left(1-\lambda^{2} / 2+\ldots\right)=0.135_{-0.016}^{+0.031}$, and $\eta\left(1-\lambda^{2} / 2+\ldots\right)=0.349_{-0.017}^{+0.015}[6]$. The parameter $\lambda$ serves as a good indicator of hierarchy of the mixing phenomenon in quark sector. Since when $\lambda$ goes to zero, the matrix $V_{\mathrm{CKM}}$ becomes a unit matrix, one can take the unit matrix as the zeroth order basis in this perturbative expansion.

The Wolfenstein parametrization is therefore an expansion of $V_{\mathrm{CKM}}$ around the unit matrix basis with $\lambda$ as the expanding parameter. The connection to the usual three angle and one phase parametrization can be identified as

$$
\lambda=s_{12} c_{13}, \quad A \lambda^{2}=s_{23} c_{13}, \quad A \lambda^{3}(\rho-i \eta)=s_{13} e^{-i \delta}
$$

One can then use this definition for the angles $\theta_{i j}$ and the phase $\delta$ to make exact parametrization of $V_{\mathrm{CKM}}$ and expand it at an arbitrary power of $\lambda$. In this type of parametrization, the choice of the parameters and where to put them are arbitrary making the meaning of the parameters subtle to some extent, for example the CP violating phase $\delta$ is not independent, i.e., it is determined by two parameters with, $\tan \delta=\eta / \rho$.

It would be better to expand the mixing matrix $V$ with the parameters kept small with clear physical meaning. One good choice is that the expanding parameters also indicate mixing in generation space. Then the procedure for finding a perturbative expanding series is to identify the zeroth order mixing matrix $V_{0}$ and then use three small mixing parameters and one $\mathrm{CP}$ violating phase to expand the mixing matrix $V$. This is the triminimal parametrization.

A good expansion is then judged by a good choice of $V_{0}$ such that the expansion converges quickly. For the quark mixing, the unit matrix is a reasonable zeroth order expansion, since the expanding parameter $\lambda=0.2257$ makes the convergence reasonably fast. But the choice of unit matrix as the zeroth order matrix $V_{0}$ is certainly not a good one for lepton sector, where it has been shown experimentally that some of the off diagonal mixing elements are not small. A direct copy of Wolfenstein parametrization for $U_{\mathrm{PMNS}}$ is not suitable. In this situation, to incorporate experimental information, it may be better to use the bimaximal mixing matrix $U_{\mathrm{bi}}[10]$ or the tri-bimaximal mixing matrix $U_{\text {tri }}$ [11] as the zeroth order basis with

$$
U_{\mathrm{bi}}=\left(\begin{array}{ccc}
1 / \sqrt{2} & 1 / \sqrt{2} & 0 \\
-1 / 2 & 1 / 2 & 1 / \sqrt{2} \\
1 / 2 & -1 / 2 & 1 / \sqrt{2}
\end{array}\right), \quad U_{\mathrm{tri}}=\left(\begin{array}{ccc}
2 / \sqrt{6} & 1 / \sqrt{3} & 0 \\
-1 / \sqrt{6} & 1 / \sqrt{3} & 1 / \sqrt{2} \\
1 / \sqrt{6} & -1 / \sqrt{3} & 1 / \sqrt{2}
\end{array}\right)
$$


Although the bimaximal basis is not favored by present experimental data, with corrections of order $\lambda$, it can accommodate experimental data, the bimaximal basis is therefore a reasonable one as good as the unit basis in quark mixing. The tri-bimaximal basis is very close to the experimental mixing pattern. It is certainly a good basis for lepton mixing expansion.

If one tries in a similar way to parameterize perturbation in expansion in a Wolfenstein-like way, there are ambiguities in how to choose the expanding parameters based on $U_{\mathrm{bi}}[12,13]$ and $U_{\text {tri }}[14$, 15]. It would be desirable to have a definitive way to make expansions. To this end, Pakvasa, Rodejohann, and Weiler proposed the triminimal parametrization in lepton sector [16]. The triminimal expansion of the quark and lepton mixing pointed out a new way to parameterize the mixing matrix with all angle parameters small, and with the CP violating phase parameter free from other parameters. The parameters are completely determined when the basis matrix is chosen.

The parametrizations of mixing for quark and lepton sectors, a priori, seems unrelated. If there is a way to connect the seemingly independent parametrizations of mixing in these two sectors, it would gain more insights about fermion mixing. Indeed there is a very nice way to make the connection via the so called quark-lepton complementarity (QLC) [17, 18]. We find the QLC relations very useful and will use it in our later discussions.

In this paper, with the help of QLC we present a detailed study of parameterizing quark and lepton mixing matrices using the triminimal parametrization technique with different basis matrices, and discuss their relations with Wolfenstein-like parametrizations.

The organization of this paper is as follows: in Sec. II, we derive the general expression of the triminimal parametrizations of the mixing matrix. In Sec. III, we take the unit matrix as the basis of the CKM matrix and the bimaximal matrix as the basis of the PMNS matrix. We show that these two parametrizations are related by QLC. In Sec. IV, we start from tri-bimaximal for lepton mixing, and then use the QLC relations to determine the basis for the CKM matrix and discuss some implications. In both Sec. III and IV, we also discuss how Wolfenstein-like parametrizations can be obtained from triminimal parametrizations. In Sec. V, we present our conclusions.

\section{THE GENERAL EXPRESSION OF THE TRIMINIMAL PARAMETRIZATIONS}

\section{A. The triminimal expansion}

The idea of the triminimal parametrization is to express a mixing angle in the mixing matrices as the sum of a zeroth order angle $\theta^{0}$ and a small perturbation angle $\epsilon$ with

$$
\theta_{12}=\theta_{12}^{0}+\epsilon_{12}, \quad \theta_{23}=\theta_{23}^{0}+\epsilon_{23}, \quad \theta_{13}=\theta_{13}^{0}+\epsilon_{13} .
$$

With the deviations $\epsilon_{12,23,13}$, one can expand the matrix elements in powers of $\epsilon_{12,23,13}$. We have

$$
V=R_{23}^{\epsilon} R_{23}^{0} U_{\delta}^{\dagger} R_{13}^{0} R_{13}^{\epsilon} U_{\delta} R_{12}^{0} R_{12}^{\epsilon},
$$

where $R_{i j}^{0}=R_{i j}\left(\theta_{i j}^{0}\right)$ and $R_{i j}^{\epsilon}=R_{i j}^{\epsilon}\left(\epsilon_{i j}\right)$.

The above can be written in a different form more suitable for expansion in $\epsilon_{i j}$,

$$
\begin{aligned}
V & =\left(R_{23}^{0}+R_{23}^{0 \prime} \sin \epsilon_{23}+2 R_{23}^{0 \prime \prime} \sin ^{2} \frac{\epsilon_{23}}{2}\right) U_{\delta}^{\dagger}\left(R_{13}^{0}+R_{13}^{0 \prime} \sin \epsilon_{13}+2 R_{13}^{0 \prime \prime} \sin ^{2} \frac{\epsilon_{13}}{2}\right) U_{\delta} \\
& \times\left(R_{12}^{0}+R_{12}^{0 \prime} \sin \epsilon_{12}+2 R_{12}^{0 \prime \prime} \sin ^{2} \frac{\epsilon_{12}}{2}\right),
\end{aligned}
$$

where $R_{i j}^{0 \prime}=\partial R_{i j}^{0} / \partial \theta_{i j}^{0}$ and $R_{i j}^{0 \prime \prime}=\partial^{2} R_{i j}^{0} / \partial\left(\theta_{i j}^{0}\right)^{2}$.

To second order in $\epsilon_{i j}$, the mixing matrix is given by

$$
\begin{aligned}
V & =V_{0}+R_{23}^{0} U_{\delta}^{\dagger} R_{13}^{0} U_{\delta} R_{12}^{0 \prime} \epsilon_{12}+R_{23}^{0 \prime} U_{\delta}^{\dagger} R_{13}^{0} U_{\delta} R_{12}^{0} \epsilon_{23}+R_{23}^{0} U_{\delta}^{\dagger} R_{13}^{0 \prime} U_{\delta} R_{12}^{0} \epsilon_{13} \\
& +\frac{1}{2} R_{23}^{0} U_{\delta}^{\dagger} R_{13}^{0} U_{\delta} R_{12}^{0 \prime \prime} \epsilon_{12}^{2}+\frac{1}{2} R_{23}^{0 \prime \prime} U_{\delta}^{\dagger} R_{13}^{0} U_{\delta} R_{12}^{0} \epsilon_{23}^{2}+\frac{1}{2} R_{23}^{0} U_{\delta}^{\dagger} R_{13}^{0 \prime \prime} U_{\delta} R_{12}^{0} \epsilon_{13}^{2} \\
& +R_{23}^{0 \prime} U_{\delta}^{\dagger} R_{13}^{0} U_{\delta} R_{12}^{0 \prime} \epsilon_{12} \epsilon_{23}+R_{23}^{0} U_{\delta}^{\dagger} R_{13}^{0 \prime} U_{\delta} R_{12}^{0 \prime} \epsilon_{12} \epsilon_{13}+R_{23}^{0 \prime} U_{\delta}^{\dagger} R_{13}^{0 \prime} U_{\delta} R_{12}^{0} \epsilon_{23} \epsilon_{13}+\mathcal{O}\left(\epsilon_{i j}^{3}\right),
\end{aligned}
$$

where $V_{0}=R_{23}^{0} U_{\delta}^{\dagger} R_{13}^{0} U_{\delta} R_{12}^{0}$ is the zeroth order expansion basis.

The Jarlskog parameter [19] $J=\operatorname{Im}\left(V_{12} V_{23} V_{13}^{*} V_{32}^{*}\right)=\frac{1}{8} \sin 2 \theta_{12} \sin 2 \theta_{23} \sin 2 \theta_{13} \cos \theta_{13} \sin \delta$ is phase-convention independent which makes it very important when discussing $\mathrm{CP}$ violation. If $\mathrm{CP}$ violation is going to be discussed, 
one should make sure that the expansion to a certain order, a non-zero $J$ is obtained. The second order in $\epsilon_{i j}$ for $J$ is given by

$$
\begin{aligned}
J & =J_{0}\left(1+2 \epsilon_{12} \cot 2 \theta_{12}^{0}+2 \epsilon_{23} \cot 2 \theta_{23}^{0}+\epsilon_{13}\left(2 \cot 2 \theta_{13}^{0}-\tan \theta_{13}^{0}\right)\right. \\
& -2 \epsilon_{12}^{2}-2 \epsilon_{23}^{2}-\epsilon_{13}^{2}\left(\frac{7}{2}-\tan ^{2} \theta_{13}^{0}\right)+4 \epsilon_{12} \epsilon_{23} \cot 2 \theta_{12}^{0} \cot 2 \theta_{23}^{0} \\
& \left.+2 \epsilon_{12} \epsilon_{13} \cot 2 \theta_{12}^{0}\left(2 \cot 2 \theta_{13}^{0}-\tan \theta_{13}^{0}\right)+2 \epsilon_{23} \epsilon_{13} \cot 2 \theta_{23}^{0}\left(2 \cot 2 \theta_{13}^{0}-\tan \theta_{13}^{0}\right)\right)+\mathcal{O}\left(\epsilon_{i j}^{3}\right),
\end{aligned}
$$

where $J_{0}=\frac{1}{8} \sin 2 \theta_{12}^{0} \sin 2 \theta_{23}^{0} \sin 2 \theta_{13}^{0} \cos \theta_{13}^{0} \sin \delta$.

Since $\theta_{13}$ in both quark and lepton sectors are very small, a good choice for $\theta_{13}^{0}$ in Eq. (11) is zero. Then Eq. (3) is simplified to

$$
R_{23}\left(\theta_{23}^{0}+\epsilon_{23}\right) U_{\delta}^{\dagger} R_{13}\left(\theta_{13}^{0}+\epsilon_{13}\right) U_{\delta} R_{12}\left(\theta_{12}^{0}+\epsilon_{12}\right)=R_{23}\left(\theta_{23}^{0}\right) R\left(\epsilon_{23}\right) U_{\delta}^{\dagger} R_{13}\left(\epsilon_{13}\right) U_{\delta} R_{12}\left(\epsilon_{12}\right) R_{12}\left(\theta_{12}^{0}\right) .
$$

Note that $R\left(\epsilon_{23}\right) U_{\delta}^{\dagger} R_{13}\left(\epsilon_{13}\right) U_{\delta} R_{12}\left(\epsilon_{12}\right)$ is just Eq. (2) with $\theta_{i j}$ replaced by $\epsilon_{i j} \cdot R_{23}\left(\theta_{23}^{0}\right) R_{12}\left(\theta_{12}^{0}\right)$ is the zeroth order approximation of the mixing matrix $V_{0}$. In this case, Eq. (14) is simplified to be

$$
\begin{aligned}
& V=\left(\begin{array}{ccc}
c_{12}^{0} c_{13}^{0} & s_{12}^{0} c_{13}^{0} & 0 \\
-s_{12}^{0} c_{23}^{0} & c_{12}^{0} c_{23}^{0} & s_{23}^{0} \\
s_{12}^{0} s_{23}^{0} & -c_{12}^{0} s_{23}^{0} & c_{23}^{0}
\end{array}\right)+\epsilon_{12}\left(\begin{array}{ccc}
-s_{12}^{0} & c_{12}^{0} & 0 \\
-c_{12}^{0} c_{23}^{0} & -s_{12}^{0} c_{23}^{0} & 0 \\
c_{12}^{0} s_{23}^{0} & s_{12}^{0} s_{23}^{0} & 0
\end{array}\right)+\epsilon_{23}\left(\begin{array}{ccc}
0 & 0 & 0 \\
s_{12}^{0} s_{23}^{0} & -c_{12}^{0} s_{23}^{0} & c_{23}^{0} \\
s_{12}^{0} c_{23}^{0} & -c_{12}^{0} c_{23}^{0} & -s_{23}^{0}
\end{array}\right) \\
& +\epsilon_{13}\left(\begin{array}{ccc}
0 & 0 & e^{-i \delta} \\
-c_{12}^{0} s_{23}^{0} e^{i \delta} & -s_{12}^{0} s_{23}^{0} e^{i \delta} & 0 \\
-c_{12}^{0} c_{23}^{0} e^{i \delta} & -s_{12}^{0} c_{23}^{0} e^{i \delta} & 0
\end{array}\right)+\frac{1}{2} \epsilon_{12}^{2}\left(\begin{array}{ccc}
-c_{12}^{0} & -s_{12}^{0} & 0 \\
s_{12}^{0} c_{23}^{0} & -c_{12}^{0} c_{23}^{0} & 0 \\
-s_{12}^{0} s_{23}^{0} & c_{12}^{0} s_{23}^{0} & 0
\end{array}\right)+\frac{1}{2} \epsilon_{23}^{2}\left(\begin{array}{ccc}
0 & 0 & 0 \\
s_{12}^{0} c_{23}^{0} & -c_{12}^{0} c_{23}^{0} & -s_{23}^{0} \\
-s_{12}^{0} s_{23}^{0} & c_{12}^{0} s_{23}^{0} & -c_{23}^{0}
\end{array}\right) \\
& +\frac{1}{2} \epsilon_{13}^{2}\left(\begin{array}{ccc}
-c_{12}^{0} & -s_{12}^{0} & 0 \\
0 & 0 & -s_{23}^{0} \\
0 & 0 & -c_{23}^{0}
\end{array}\right)+\epsilon_{12} \epsilon_{23}\left(\begin{array}{ccc}
0 & 0 & 0 \\
c_{12}^{0} s_{23}^{0} & s_{12}^{0} s_{23}^{0} & 0 \\
c_{12}^{0} c_{23}^{0} & s_{12}^{0} c_{23}^{0} & 0
\end{array}\right)+\epsilon_{12} \epsilon_{13}\left(\begin{array}{ccc}
0 & 0 & 0 \\
s_{12}^{0} s_{23}^{0} e^{i \delta} & -c_{12}^{0} s_{23}^{0} e^{i \delta} & 0 \\
s_{12}^{0} c_{23}^{0} e^{i \delta} & -c_{12}^{0} c_{23}^{0} e^{i \delta} & 0
\end{array}\right) \\
& +\epsilon_{23} \epsilon_{13}\left(\begin{array}{ccc}
0 & 0 & 0 \\
-c_{12}^{0} c_{23}^{0} e^{i \delta} & -s_{12}^{0} c_{23}^{0} e^{i \delta} & 0 \\
c_{12}^{0} s_{23}^{0} e^{i \delta} & s_{12}^{0} s_{23}^{0} e^{i \delta} & 0
\end{array}\right)+\mathcal{O}\left(\epsilon_{i j}^{3}\right) \text {. }
\end{aligned}
$$

The Jarlskog parameter $J$ is then given by

$$
J=\left(\epsilon_{13} c_{12}^{0} c_{23}^{0} s_{12}^{0} s_{23}^{0}+\frac{1}{2} \epsilon_{12} \epsilon_{13} \sin 2 \theta_{23}^{0} \cos 2 \theta_{12}^{0}+\frac{1}{2} \epsilon_{23} \epsilon_{13} \sin 2 \theta_{12}^{0} \cos 2 \theta_{23}^{0}\right) \sin \delta+O\left(\epsilon_{i j}^{3}\right) .
$$

It is clear from above discussions that in general the expansion looks complicated. So far the discussions are just a simple expansion in mathematics. A good expansion should have the virtual being simple with fast convergency. The choice of the zeroth order matrix $V_{0}$ which leads to a simple expansion then reflects the physical insight of a parametrization providing hints for underlying theory producing the mixing.

\section{B. Unified parametrization through quark-lepton complementarity}

So far we have treated the parametrizations for quarks and leptons separately. It would be interesting to find a unified parametrization which connects these two seemingly unrelated sectors. We find that the quark-lepton complementarity (QLC) can provide a very useful guide for the unified treatment of mixing in quark and lepton sectors. The QLC relations refer to

$$
\theta_{12}^{Q}+\theta_{12}^{L}=\frac{\pi}{4}, \quad \theta_{23}^{Q}+\theta_{23}^{L}=\frac{\pi}{4}, \quad \theta_{13}^{Q} \sim \theta_{13}^{L} \sim 0
$$

If one writes

$$
\begin{array}{lll}
\theta_{12}^{Q}=\theta_{12}^{Q 0}+\epsilon_{12}^{Q}, & \theta_{23}^{Q}=\theta_{23}^{Q 0}+\epsilon_{23}^{Q}, & \theta_{13}^{Q}=\theta_{13}^{Q 0}+\epsilon_{13}^{Q}, \\
\theta_{12}^{L}=\theta_{12}^{L 0}+\epsilon_{12}^{L}, & \theta_{23}^{L}=\theta_{23}^{L 0}+\epsilon_{23}^{L}, & \theta_{13}^{L}=\theta_{13}^{L 0}+\epsilon_{13}^{L},
\end{array}
$$

a good choice of the zeroth order angles would be: $\theta_{12}^{Q 0}+\theta_{12}^{L 0}=\pi / 4, \theta_{23}^{Q 0}+\theta_{23}^{L 0}=\pi / 4$, and $\theta_{13}^{Q 0}=\theta_{13}^{L 0}=0$. One has

$$
\epsilon_{12}^{Q}+\epsilon_{12}^{L}=0, \quad \epsilon_{23}^{Q}+\epsilon_{23}^{L}=0, \quad \epsilon_{13}^{Q} \sim \epsilon_{13}^{L} \sim 0 .
$$


This procedure then leads to

$$
\begin{aligned}
V_{\mathrm{CKM}} & =R_{23}\left(\theta_{23}^{Q 0}\right) R_{23}\left(\epsilon_{23}^{Q}\right) U_{\delta}^{\dagger} R_{13}\left(\epsilon_{13}^{Q}\right) U_{\delta} R_{12}\left(\epsilon_{12}^{Q}\right) R_{12}\left(\theta_{12}^{0}\right) \\
U_{\mathrm{PMNS}} & =R_{23}\left(\frac{\pi}{4}-\theta_{23}^{Q 0}\right) R_{23}\left(\epsilon_{23}^{L}\right) U_{\delta}^{\dagger} R_{13}\left(\epsilon_{13}^{L}\right) U_{\delta} R_{12}\left(\epsilon_{12}^{L}\right) R_{12}\left(\frac{\pi}{4}-\theta_{12}^{Q 0}\right) \\
& =R_{23}\left(\frac{\pi}{4}\right) R_{23}\left(-\theta_{23}^{Q 0}\right) R_{23}\left(-\epsilon_{23}^{Q}\right) U_{\delta}^{\dagger} R_{13}\left(\epsilon_{13}^{L}\right) U_{\delta} R_{12}\left(-\epsilon_{12}^{Q}\right) R_{12}\left(-\theta_{12}^{Q 0}\right) R_{12}\left(\frac{\pi}{4}\right) .
\end{aligned}
$$

The corresponding Jarlskog parameters are also related,

$$
\begin{aligned}
J^{Q} & =\frac{1}{4} \sin 2 \theta_{12}^{Q} \sin 2 \theta_{23}^{Q} \sin \epsilon_{13}^{Q} \cos ^{2} \epsilon_{13}^{Q} \sin \delta^{Q}, \\
J^{L} & =\frac{1}{4} \cos 2 \theta_{12}^{Q} \cos 2 \theta_{23}^{Q} \sin \epsilon_{13}^{L} \cos ^{2} \epsilon_{13}^{L} \sin \delta^{L} .
\end{aligned}
$$

The above relations reveal an important difference in CP violation in the quark and lepton sectors. Since phenomenologically, $\theta_{12}^{Q}$ and $\theta_{23}^{Q}$ are both small, it is clear that the absolute value of $J^{L}$ is much larger than the absolute value of $J^{Q}$ if $\epsilon_{13}^{L}$ and $\delta^{L}$ are not much smaller than $\epsilon_{13}^{Q}$ and $\delta^{Q}$.

In the following sections, we work out some specific choices of $V_{0}$ and compare early studies with the triminimal parametrization we are discussing here.

\section{TRIMINIMAL PARAMETRIZATIONS OF THE CKM AND PMNS MATRICES WITH UNIT AND BIMAXIMAL BASIS MATRICES}

\section{A. The triminimal expansion}

Eq. (5) shows that the off-diagonal elements in the CKM matrix are all small compared with 1, it is natural to take the unit matrix as its basis. Setting the expanding triminimal parameters as

$$
\theta_{12}^{Q}=\epsilon_{12}^{Q}, \quad \theta_{23}^{Q}=\epsilon_{23}^{Q}, \quad \theta_{13}^{Q}=\epsilon_{13}^{Q},
$$

and the CP-violating phase $\delta^{Q}$ for quark, we obtain the triminimal parametrization of the CKM matrix as

$$
V_{\mathrm{CKM}}=R_{23}\left(\epsilon_{23}^{Q}\right) U_{\delta}^{\dagger} R_{13}\left(\epsilon_{13}^{Q}\right) U_{\delta} R_{12}\left(\epsilon_{12}^{Q}\right) .
$$

Numerically, we have

$$
\epsilon_{12}^{Q}=0.2277 \pm 0.0010, \quad \epsilon_{23}^{Q}=0.0415_{-0.0011}^{+0.0010}, \quad \epsilon_{13}^{Q}=0.00359 \pm 0.00016 .
$$

To third order in $\epsilon_{i j}^{Q}$, we have

$$
\begin{aligned}
V_{\mathrm{CKM}} & =I+\left(\begin{array}{lll}
0 & \epsilon_{12}^{Q} & \epsilon_{13}^{Q} e^{-i \delta^{Q}} \\
-\epsilon_{12}^{Q} & 0 & \epsilon_{23}^{Q} \\
-\epsilon_{13}^{Q} e^{i \delta^{Q}} & -\epsilon_{23}^{Q} & 0
\end{array}\right)+\left(\begin{array}{ccc}
-\frac{1}{2}\left(\epsilon_{12}^{Q}\right)^{2}-\frac{1}{2}\left(\epsilon_{13}^{Q}\right)^{2} & 0 & 0 \\
-\epsilon_{23}^{Q} \epsilon_{13}^{Q} e^{i \delta^{Q}} & -\frac{1}{2}\left(\epsilon_{12}^{Q}\right)^{2}-\frac{1}{2}\left(\epsilon_{23}^{Q}\right)^{2} & 0 \\
\epsilon_{12}^{Q} \epsilon_{23}^{Q} & -\epsilon_{12}^{Q} \epsilon_{13}^{Q} e^{i \delta^{Q}} & -\frac{1}{2}\left(\epsilon_{23}^{Q}\right)^{2}-\frac{1}{2}\left(\epsilon_{13}^{Q}\right)^{2}
\end{array}\right) \\
& +\left(\begin{array}{ccc}
0 & -\frac{1}{6}\left(\epsilon_{12}^{Q}\right)^{3}-\frac{1}{2} \epsilon_{12}^{Q}\left(\epsilon_{13}^{Q}\right)^{2} & -\frac{1}{6}\left(\epsilon_{13}^{Q}\right)^{3} e^{-i \delta^{Q}} \\
\frac{1}{6}\left(\epsilon_{12}^{Q}\right)^{3}+\frac{1}{2} \epsilon_{12}^{Q}\left(\epsilon_{23}^{Q}\right)^{2} & -\epsilon_{12}^{Q} \epsilon_{23}^{Q} \epsilon_{13}^{Q} e^{i \delta^{Q}} & -\frac{1}{6}\left(\epsilon_{23}^{Q}\right)^{3}-\frac{1}{2} \epsilon_{23}^{Q}\left(\epsilon_{13}^{Q}\right)^{2} \\
\frac{1}{2}\left(\epsilon_{12}^{Q}\right)^{2} \epsilon_{13}^{Q} e^{i \delta^{Q}}+\frac{1}{2}\left(\epsilon_{23}^{Q}\right)^{2} \epsilon_{13}^{Q} e^{i \delta^{Q}} & \frac{1}{6}\left(\epsilon_{23}^{Q}\right)^{3}+\frac{1}{2}\left(\epsilon_{12}^{Q}\right)^{2} \epsilon_{23}^{Q} & 0
\end{array}\right)+\mathcal{O}\left(\left(\epsilon_{i j}^{Q}\right)^{4}\right),
\end{aligned}
$$

where $I$ denotes the $3 \times 3$ unit matrix.

In most cases, the approximation to second order in $\epsilon_{i j}^{Q}$ is enough. Here we keep it to the third order because that $\epsilon_{13}^{Q}$ is of order $\left(\epsilon_{12}^{Q}\right)^{3}$, for numerical consistency one should display terms of order $\left(\epsilon_{12}^{Q}\right)^{3}$ in the expansion if an expansion involves $\epsilon_{13}^{Q}$. Also since $\epsilon_{23}^{Q}$ is of order $\left(\epsilon_{12}^{Q}\right)^{2}$, one should keep $\epsilon_{12}^{Q} \epsilon_{23}^{Q}$ in the expansion. We then have a numerical consistent expansion

$$
\begin{aligned}
V_{\mathrm{CKM}} & =I+\left(\begin{array}{ccc}
0 & \epsilon_{12}^{Q} & \epsilon_{13}^{Q} e^{-i \delta^{Q}} \\
-\epsilon_{12}^{Q} & 0 & \epsilon_{23}^{Q} \\
-\epsilon_{13}^{Q} e^{i \delta^{Q}} & -\epsilon_{23}^{Q} & 0
\end{array}\right)+\left(\begin{array}{ccc}
-\frac{1}{2}\left(\epsilon_{12}^{Q}\right)^{2} & 0 & 0 \\
0 & -\frac{1}{2}\left(\epsilon_{12}^{Q}\right)^{2} & 0 \\
\epsilon_{12}^{Q} \epsilon_{23}^{Q} & 0 & 0
\end{array}\right) \\
& +\left(\begin{array}{ccc}
0 & -\frac{1}{6}\left(\epsilon_{12}^{Q}\right)^{3} & 0 \\
\frac{1}{6}\left(\epsilon_{12}^{Q}\right)^{3} & 0 & 0 \\
0 & 0 & 0
\end{array}\right)+\mathcal{O}\left(\left(\epsilon_{12}^{Q}\right)^{4}\right) .
\end{aligned}
$$


For lepton mixing matrix, if one use QLC as a guide, the corresponding expansion in trimnimal expansion would give

$$
U_{\mathrm{PMNS}}=R_{23}\left(\frac{\pi}{4}\right) R_{23}\left(\epsilon_{23}^{L}\right) U_{\delta}^{\dagger} R_{13}\left(\epsilon_{13}^{L}\right) U_{\delta} R_{12}\left(\epsilon_{12}^{L}\right) R_{12}\left(\frac{\pi}{4}\right)
$$

with $\epsilon_{12,23}^{L}=-\epsilon_{12,23}^{Q}$.

Numerically, the $1 \sigma(3 \sigma)$ ranges of the triminimal parameters are

$$
(-0.25)-0.21<\epsilon_{12}^{L}<-0.16(-0.10), \quad(-0.18)-0.10<\epsilon_{23}^{L}<0.04(0.15), \quad(0.00) 0.00<\epsilon_{13}^{L}<0.14(0.23) .
$$

with the best fit values $\epsilon_{12}^{L}=-0.18, \epsilon_{23}^{L}=-0.05$ and $\epsilon_{13}^{L}=0.00$. Experimental data are consistent with QLC at $3 \sigma$ level. Should future experimental data determine that QLC is inconsistent with data, one will have to abandon QLC, but the expansion using bimaximal mixing matrix $U_{b i}$ is still valid and can serve as one of the expansions of the PMNS matrix.

To third order in $\epsilon_{i j}^{L}$, the expansion is

$$
\begin{aligned}
& U_{\mathrm{PMNS}}=U_{\mathrm{bi}}+\epsilon_{12}^{L}\left(\begin{array}{ccc}
-\frac{\sqrt{2}}{2} & \frac{\sqrt{2}}{2} & 0 \\
-\frac{1}{2} & -\frac{1}{2} & 0 \\
\frac{1}{2} & \frac{1}{2} & 0
\end{array}\right)+\epsilon_{23}^{L}\left(\begin{array}{ccc}
0 & 0 & 0 \\
\frac{1}{2} & -\frac{1}{2} & \frac{\sqrt{2}}{2} \\
\frac{1}{2} & -\frac{1}{2} & -\frac{\sqrt{2}}{2}
\end{array}\right)+\epsilon_{13}^{L}\left(\begin{array}{ccc}
0 & 0 & e^{-i \delta^{L}} \\
-\frac{1}{2} e^{i \delta^{L}} & -\frac{1}{2} e^{i \delta^{L}} & 0 \\
-\frac{1}{2} e^{i \delta^{L}} & -\frac{1}{2} e^{i \delta^{L}} & 0
\end{array}\right) \\
& +\left(\epsilon_{12}^{L}\right)^{2}\left(\begin{array}{ccc}
-\frac{\sqrt{2}}{4} & -\frac{\sqrt{2}}{4} & 0 \\
\frac{1}{4} & -\frac{1}{4} & 0 \\
-\frac{1}{4} & \frac{1}{4} & 0
\end{array}\right)+\left(\epsilon_{23}^{L}\right)^{2}\left(\begin{array}{ccc}
0 & 0 & 0 \\
\frac{1}{4} & -\frac{1}{4} & -\frac{\sqrt{2}}{4} \\
-\frac{1}{4} & \frac{1}{4} & -\frac{\sqrt{2}}{4}
\end{array}\right)+\left(\epsilon_{13}^{L}\right)^{2}\left(\begin{array}{ccc}
-\frac{\sqrt{2}}{4} & -\frac{\sqrt{2}}{4} & 0 \\
0 & 0 & -\frac{\sqrt{2}}{4} \\
0 & 0 & -\frac{\sqrt{2}}{4}
\end{array}\right) \\
& +\epsilon_{12}^{L} \epsilon_{23}^{L}\left(\begin{array}{ccc}
0 & 0 & 0 \\
\frac{1}{2} & \frac{1}{2} & 0 \\
\frac{1}{2} & \frac{1}{2} & 0
\end{array}\right)+\epsilon_{12}^{L} \epsilon_{13}^{L} e^{i \delta^{L}}\left(\begin{array}{ccc}
0 & 0 & 0 \\
\frac{1}{2} & -\frac{1}{2} & 0 \\
\frac{1}{2} & -\frac{1}{2} & 0
\end{array}\right)+\epsilon_{23}^{L} \epsilon_{13}^{L} e^{i \delta^{L}}\left(\begin{array}{ccc}
0 & 0 & 0 \\
-\frac{1}{2} & -\frac{1}{2} & 0 \\
\frac{1}{2} & \frac{1}{2} & 0
\end{array}\right) \\
& +\left(\epsilon_{12}^{L}\right)^{3}\left(\begin{array}{ccc}
\frac{\sqrt{2}}{12} & -\frac{\sqrt{2}}{12} & 0 \\
\frac{1}{12} & \frac{1}{12} & 0 \\
-\frac{1}{12} & -\frac{1}{12} & 0
\end{array}\right)+\left(\epsilon_{23}^{L}\right)^{3}\left(\begin{array}{ccc}
0 & 0 & 0 \\
-\frac{1}{12} & \frac{1}{12} & -\frac{\sqrt{2}}{12} \\
-\frac{1}{12} & \frac{1}{12} & \frac{\sqrt{2}}{12}
\end{array}\right)+\left(\epsilon_{13}^{L}\right)^{3}\left(\begin{array}{ccc}
0 & 0 & -\frac{1}{6} e^{-i \delta^{L}} \\
\frac{1}{12} e^{i \delta^{L}} & \frac{1}{12} e^{i \delta^{L}} & 0 \\
\frac{1}{12} e^{i \delta^{L}} & \frac{1}{12} e^{i \delta^{L}} & 0
\end{array}\right) \\
& +\epsilon_{12}^{L}\left(\epsilon_{23}^{L}\right)^{2}\left(\begin{array}{ccc}
0 & 0 & 0 \\
\frac{1}{4} & \frac{1}{4} & 0 \\
-\frac{1}{4} & -\frac{1}{4} & 0
\end{array}\right)+\left(\epsilon_{12}^{L}\right)^{2} \epsilon_{23}^{L}\left(\begin{array}{ccc}
0 & 0 & 0 \\
-\frac{1}{4} & \frac{1}{4} & 0 \\
-\frac{1}{4} & \frac{1}{4} & 0
\end{array}\right)+\epsilon_{12}^{L}\left(\epsilon_{13}^{L}\right)^{2}\left(\begin{array}{ccc}
\frac{\sqrt{2}}{4} & -\frac{\sqrt{2}}{4} & 0 \\
0 & 0 & 0 \\
0 & 0 & 0
\end{array}\right) \\
& +\left(\epsilon_{12}^{L}\right)^{2} \epsilon_{13}^{L} e^{i \delta^{L}}\left(\begin{array}{ccc}
0 & 0 & 0 \\
\frac{1}{4} & \frac{1}{4} & 0 \\
\frac{1}{4} & \frac{1}{4} & 0
\end{array}\right)+\epsilon_{23}^{L}\left(\epsilon_{13}^{L}\right)^{2}\left(\begin{array}{ccc}
0 & 0 & 0 \\
0 & 0 & -\frac{\sqrt{2}}{4} \\
0 & 0 & \frac{\sqrt{2}}{4}
\end{array}\right)+\left(\epsilon_{23}^{L}\right)^{2} \epsilon_{13}^{L} e^{i \delta^{L}}\left(\begin{array}{ccc}
0 & 0 & 0 \\
\frac{1}{4} & \frac{1}{4} & 0 \\
\frac{1}{4} & \frac{1}{4} & 0
\end{array}\right) \\
& +\epsilon_{12}^{L} \epsilon_{23}^{L} \epsilon_{13}^{L} e^{i \delta^{L}}\left(\begin{array}{ccc}
0 & 0 & 0 \\
\frac{1}{2} & -\frac{1}{2} & 0 \\
-\frac{1}{2} & \frac{1}{2} & 0
\end{array}\right)+\mathcal{O}\left(\left(\epsilon_{i j}^{L}\right)^{4}\right) .
\end{aligned}
$$

If $\epsilon_{i j}^{L}$ exhibit the same hierarchy as those in quark sector, that is, $\epsilon_{23}^{L} \sim\left(\epsilon_{12}^{L}\right)^{2}, \epsilon_{13}^{L} \sim\left(\epsilon_{12}^{L}\right)^{3}$, then to third order in $\epsilon_{12}^{L}$, we have

$$
\begin{aligned}
U_{\mathrm{PMNS}} & =U_{\mathrm{bi}}+\epsilon_{12}^{L}\left(\begin{array}{ccc}
-\frac{\sqrt{2}}{2} & \frac{\sqrt{2}}{2} & 0 \\
-\frac{1}{2} & -\frac{1}{2} & 0 \\
\frac{1}{2} & \frac{1}{2} & 0
\end{array}\right)+\epsilon_{23}^{L}\left(\begin{array}{ccc}
0 & 0 & 0 \\
\frac{1}{2} & -\frac{1}{2} & \frac{\sqrt{2}}{2} \\
\frac{1}{2} & -\frac{1}{2} & -\frac{\sqrt{2}}{2}
\end{array}\right)+\epsilon_{13}^{L}\left(\begin{array}{ccc}
0 & 0 & e^{-i \delta^{L}} \\
-\frac{1}{2} e^{i \delta^{L}} & -\frac{1}{2} e^{i \delta^{L}} & 0 \\
-\frac{1}{2} e^{i \delta^{L}} & -\frac{1}{2} e^{i \delta^{L}} & 0
\end{array}\right) \\
& +\left(\epsilon_{12}^{L}\right)^{2}\left(\begin{array}{ccc}
-\frac{\sqrt{2}}{4} & -\frac{\sqrt{2}}{4} & 0 \\
\frac{1}{4} & -\frac{1}{4} & 0 \\
-\frac{1}{4} & \frac{1}{4} & 0
\end{array}\right)+\epsilon_{12}^{L} \epsilon_{23}^{L}\left(\begin{array}{ccc}
0 & 0 & 0 \\
\frac{1}{2} & \frac{1}{2} & 0 \\
\frac{1}{2} & \frac{1}{2} & 0
\end{array}\right)+\left(\epsilon_{12}^{L}\right)^{3}\left(\begin{array}{ccc}
\frac{\sqrt{2}}{12} & -\frac{\sqrt{2}}{12} & 0 \\
\frac{1}{12} & \frac{1}{12} & 0 \\
-\frac{1}{12} & -\frac{1}{12} & 0
\end{array}\right)+\mathcal{O}\left(\left(\epsilon_{12}^{L}\right)^{4}\right) .
\end{aligned}
$$

It is interesting to note that the basis of the PMNS matrix is naturally the bimaximal mixing matrix $V_{0}=U_{b i}$.

\section{B. Triminimal and Wolfenstein-like parametrizations}

We now compare the triminimal parametrization with Wolfenstein parametrization for quark mixing. To obtain the Wolfenstein parametrization, one may naively make the replacements of $\epsilon_{12}^{Q}=\lambda, \epsilon_{23}^{Q}=A \lambda^{2}$, and $\epsilon_{13}^{Q} e^{i \delta^{Q}}=A \lambda^{3}(\rho+i \eta)$, 
which lead to

$$
\epsilon_{13}^{Q}=A \lambda^{3} \sqrt{\rho^{2}+\eta^{2}}, \quad \epsilon_{12}^{Q} \epsilon_{23}^{Q}=A \lambda^{3} .
$$

To third order in $\lambda$, one obtains

$$
V_{\mathrm{CKM}}=\left(\begin{array}{ccc}
1-\frac{1}{2} \lambda^{2} & \lambda & A \lambda^{3}(\rho-i \eta) \\
-\lambda & 1-\frac{1}{2} \lambda^{2} & A \lambda^{2} \\
A \lambda^{3}(1-\rho-i \eta) & -A \lambda^{2} & 1
\end{array}\right)+\lambda^{3}\left(\begin{array}{ccc}
0 & -\frac{1}{6} & 0 \\
\frac{1}{6} & 0 & 0 \\
0 & 0 & 0
\end{array}\right)+\mathcal{O}\left(\lambda^{4}\right) .
$$

The first term in the above is the Wolfenstein parametrization. But there is an additional second term in the above equation. One may wonder that the triminimal parametrization may not be equivalent to the Wolfenstein parametrization. This is certainly not true. The problem lies in the definition for $\lambda$. In the Wolfenstein parametrization, $\lambda$ is defined through

$$
V_{u s}=\cos \theta_{13}^{Q} \sin \theta_{12}^{Q}=\lambda .
$$

But in the above naive identification of triminimal parametrization with Wolfenstein parametrization, $\lambda$ is defined by

$$
\lambda=\epsilon_{12}^{Q}
$$

The above two definitions of $\lambda$ 's are actually different. Let us indicate temporarily by $\lambda^{\prime}=\epsilon_{12}^{Q}$, then to third order in $\lambda$ or $\lambda^{\prime}$,

$$
\lambda=\sin \theta_{12}^{Q}=\epsilon_{12}^{Q}-\frac{1}{6}\left(\epsilon_{12}^{Q}\right)^{3}=\lambda^{\prime}-\frac{1}{6}\left(\lambda^{\prime}\right)^{3}
$$

Therefore $\lambda^{\prime}$ is larger than $\lambda$ by a correct of order $\lambda^{3}$.

To correctly pass the triminimal parametrization to the usual Wolfenstein parametrization, one needs to define

$$
\epsilon_{12}^{Q}=\lambda+\frac{1}{6} \lambda^{3}+\mathcal{O}\left(\lambda^{4}\right), \quad \epsilon_{23}^{Q}=A \lambda^{2}+\mathcal{O}\left(\lambda^{4}\right), \quad \epsilon_{13}^{Q} e^{i \delta^{Q}}=A \lambda^{3}(\rho+i \eta)+\mathcal{O}\left(\lambda^{4}\right) .
$$

With these definitions, the triminimal parametrization can be written in the same form as the Wolfenstein parametrization with the same order of accuracy.

Now let us consider the mixing in the lepton sector seeking the corresponding parametrization of the Wolfensteinlike parametrization. In the quark sector, the largest triminimal expansion parameter is $\epsilon_{12}^{Q}=\lambda$. One may start with a similar set of expanding parameters with the replacements $\epsilon_{12}^{L}=-\lambda, \epsilon_{23}^{L}=-A \lambda^{2}$, and $\epsilon_{13}^{L} e^{i \delta^{L}}=A \lambda^{3}(\zeta+i \xi)$, then

$$
\epsilon_{13}^{L}=A \lambda^{3} \sqrt{\zeta^{2}+\xi^{2}}, \quad \epsilon_{12}^{L} \epsilon_{23}^{L}=A \lambda^{3} .
$$

Comparing with the parametrization of Case $1\left(\epsilon_{13}^{L}=\left|U_{e 3}\right| \sim \lambda^{3} \sim\left(\epsilon_{12}^{L}\right)^{3}\right)$ in Ref. [13],

$$
\begin{aligned}
U & =U_{\mathrm{bi}}+\lambda\left(\begin{array}{ccc}
\frac{\sqrt{2}}{2} & -\frac{\sqrt{2}}{2} & 0 \\
\frac{1}{2} & \frac{1}{2} & 0 \\
-\frac{1}{2} & -\frac{1}{2} & 0
\end{array}\right)+\lambda^{2}\left(\begin{array}{ccc}
-\frac{\sqrt{2}}{4} & -\frac{\sqrt{2}}{4} & 0 \\
-\frac{1}{2}\left(A-\frac{1}{2}\right) & \frac{1}{2}\left(A-\frac{1}{2}\right) & -\frac{\sqrt{2}}{\sqrt{2}} A \\
-\frac{1}{2}\left(A+\frac{1}{2}\right) & \frac{1}{2}\left(A+\frac{1}{2}\right) & \frac{\sqrt{2}}{2} A
\end{array}\right) \\
& +\lambda^{3}\left(\begin{array}{ccc}
0 & 0 & A(\zeta-i \xi) \\
\frac{1}{2} A(1-\zeta-i \xi) & \frac{1}{2} A(1-\zeta-i \xi) & 0 \\
\frac{1}{2} A(1-\zeta-i \xi) & \frac{1}{2} A(1-\zeta-i \xi) & 0
\end{array}\right)+\mathcal{O}\left(\lambda^{4}\right),
\end{aligned}
$$

there is an additional term result from Eq. (32) given by

$$
\lambda^{3}\left(\begin{array}{ccc}
-\frac{\sqrt{2}}{12} & \frac{\sqrt{2}}{12} & 0 \\
-\frac{1}{12} & -\frac{1}{12} & 0 \\
\frac{1}{12} & \frac{1}{12} & 0
\end{array}\right)
$$

This difference is again caused by different definition of $\lambda$. In Ref. [13] it is defined as (to order $\lambda^{3}$ )

$$
\sin \theta_{12}^{L}=\frac{\sqrt{2}}{2}\left(1-\lambda-\frac{1}{2} \lambda^{2}\right) .
$$


However, in our triminimal parametrization $\lambda=-\epsilon_{12}^{L}$ is defined as (to order $\left(\epsilon_{12}^{L}\right)^{3}$ )

$$
\sin \theta_{12}^{L}=\frac{\sqrt{2}}{2}\left(1+\epsilon_{12}^{L}-\frac{1}{2}\left(\epsilon_{12}^{L}\right)^{2}-\frac{1}{6}\left(\epsilon_{12}^{L}\right)^{3}\right) .
$$

If one replaces $\epsilon_{12}^{L}$ by $-\lambda-\frac{1}{6} \lambda^{3}+\mathcal{O}\left(\lambda^{4}\right), \epsilon_{23}^{L}$ by $-A \lambda^{2}+\mathcal{O}\left(\lambda^{4}\right)$, and $\epsilon_{13}^{L} e^{i \delta^{L}}$ by $A \lambda^{3}(\zeta+i \xi)+\mathcal{O}\left(\lambda^{4}\right)$, the resulting mixing matrix from triminimal expansion give the same one as in eq.(40). The two Wolfenstein-like parametrizations in Ref. [13] and the one resulting from triminimal parametrization are equivalent.

If $\left|U_{e 3}\right|$ is not very small, but with $\epsilon_{13}^{L} \sim\left(\epsilon_{12}^{L}\right)^{2}$, which is the Case 2 discussed in Ref. [13], then to third order in $\epsilon_{12}^{L}$, we will need to keep in Eq. (32) the $\epsilon_{12}^{L} \epsilon_{13}^{L}$ term in Eq. (31). In the parametrization of Case $2\left(\epsilon_{13}^{L}=\left|U_{e 3}\right| \sim \lambda^{2} \sim\left(\epsilon_{12}^{L}\right)^{2}\right)$ in Ref. [13], the expansion of the PMNS matrix is

$$
\begin{aligned}
U & =U_{\mathrm{bi}}+\lambda\left(\begin{array}{ccc}
\frac{\sqrt{2}}{2} & -\frac{\sqrt{2}}{2} & 0 \\
\frac{1}{2} & \frac{1}{2} & 0 \\
-\frac{1}{2} & -\frac{1}{2} & 0
\end{array}\right)+\lambda^{2}\left(\begin{array}{ccc}
-\frac{\sqrt{2}}{4} & -\frac{\sqrt{2}}{4} & A\left(\zeta^{\prime}-i \xi^{\prime}\right) \\
\frac{1}{2}\left(\frac{1}{2}-A\left(1+\zeta^{\prime}+i \xi^{\prime}\right)\right) & -\frac{1}{2}\left(\frac{1}{2}-A\left(1-\zeta^{\prime}-i \xi^{\prime}\right)\right) & -\frac{\sqrt{2}}{2} A \\
-\frac{1}{2}\left(\frac{1}{2}+A\left(1+\zeta^{\prime}+i \xi^{\prime}\right)\right) & \frac{1}{2}\left(\frac{1}{2}+A\left(1-\zeta^{\prime}-i \xi^{\prime}\right)\right) & \frac{\sqrt{2}}{2} A
\end{array}\right) \\
& +\mathcal{O}\left(\lambda^{3}\right) .
\end{aligned}
$$

Making the replacements $\epsilon_{12}^{L}=-\lambda, \epsilon_{23}^{L}=-A \lambda^{2}$, and $\epsilon_{13}^{L} e^{i \delta^{L}}=A \lambda^{2}\left(\zeta^{\prime}+i \xi^{\prime}\right)$ which means

$$
\epsilon_{13}^{L}=A \lambda^{2} \sqrt{\zeta^{\prime 2}+\xi^{\prime 2}}, \quad \epsilon_{12}^{L} \epsilon_{23}^{L}=A \lambda^{3},
$$

then Eq. (32) is translated into Eq. (44). Since the expansion of Eq. (44) is an approximation to $\mathcal{O}\left(\lambda^{2}\right)$, the terms of $\left(\epsilon_{12}^{L}\right)^{3}, \epsilon_{12}^{L} \epsilon_{23}^{L}$ and $\epsilon_{12}^{L} \epsilon_{13}^{L}$ in Eq. (32) do not need to taken into account as $\mathcal{O}\left(\lambda^{3}\right)$.

To the lowest order the Jarlskog parameter are given by

$$
\begin{aligned}
& \mathcal{J}^{Q}=\operatorname{Im}\left(V_{u s} V_{c b} V_{u b}^{*} V_{c s}^{*}\right)=\epsilon_{12}^{Q} \epsilon_{23}^{Q} \epsilon_{13}^{Q} \sin \delta^{Q}, \\
& \mathcal{J}^{L}=\operatorname{Im}\left(U_{e 2} U_{\mu 3} U_{e 3}^{*} U_{\mu 2}^{*}\right)=\frac{1}{4} \epsilon_{13}^{L} \sin \delta^{L} .
\end{aligned}
$$

The two results are very simple and may be useful in experimental analysis. The expression of $\mathcal{J}^{L}$ is consistent with that in Ref. [13] to lowest order in $\lambda$.

We end this section with the conclusions that one can derive the Wolfenstein parametrization, and the Wolfensteinlike parametrization of the PMNS matrix in Ref. [13] from the triminimal parametrization in basis of the unit matrix and the bimaximal matrix. The parametrization of the PMNS matrix in Ref. [13] is unified with the Wolfenstein parametrization with the same parameters $\lambda$ and $A$ using the QLC relations. The QLC relates unit matrix basis in quark sector to the bimaximal basis in lepton sector.

\section{TRIMINIMAL PARAMETRIZATIONS OF THE CKM AND PMNS MATRICES IN TRI-BIMAXIMAL PATTERN}

\section{A. The triminimal expansion}

It is interesting to note that starting with unit matrix for $V_{0}$ for quark mixing expansion, QLC leads to bimaximal mixing matrix for $V_{0}$ for lepton sector. Present data, however, indicate that the mixing in the lepton sector is closer to the tri-bimaximal mixing. One would have a much faster convergence if an expansion for lepton sector starts with $V_{0}$ which has a tri-bimaximal mixing form.

In Ref. [16], the triminimal parametrization of the PMNS matrix is obtained as

$$
\begin{aligned}
& U_{\mathrm{PMNS}}=U_{\mathrm{tri}}+\epsilon_{12}^{L}\left(\begin{array}{ccc}
-\frac{1}{\sqrt{3}} & \frac{2}{\sqrt{6}} & 0 \\
-\frac{1}{\sqrt{3}} & -\frac{1}{\sqrt{6}} & 0 \\
\frac{1}{\sqrt{3}} & \frac{1}{\sqrt{6}} & 0
\end{array}\right)+\epsilon_{23}^{L}\left(\begin{array}{ccc}
0 & 0 & 0 \\
\frac{1}{\sqrt{6}} & -\frac{1}{\sqrt{3}} & \frac{1}{\sqrt{2}} \\
\frac{1}{\sqrt{6}} & -\frac{1}{\sqrt{3}} & -\frac{1}{\sqrt{2}}
\end{array}\right)+\epsilon_{13}^{L}\left(\begin{array}{ccc}
0 & 0 & e^{-i \delta^{L}} \\
-\frac{1}{\sqrt{3}} e^{i \delta^{L}} & -\frac{1}{\sqrt{6}} e^{i \delta^{L}} & 0 \\
-\frac{1}{\sqrt{3}} e^{i \delta^{L}} & -\frac{1}{\sqrt{6}} e^{i \delta^{L}} & 0
\end{array}\right) \\
& +\left(\epsilon_{12}^{L}\right)^{2}\left(\begin{array}{ccc}
-\frac{1}{\sqrt{6}} & -\frac{1}{2 \sqrt{3}} & 0 \\
\frac{1}{2 \sqrt{6}} & -\frac{1}{2 \sqrt{3}} & 0 \\
-\frac{1}{2 \sqrt{6}} & \frac{1}{2 \sqrt{3}} & 0
\end{array}\right)+\left(\epsilon_{23}^{L}\right)^{2}\left(\begin{array}{ccc}
0 & 0 & 0 \\
\frac{1}{2 \sqrt{6}} & -\frac{1}{2 \sqrt{3}} & -\frac{1}{2 \sqrt{2}} \\
-\frac{1}{2 \sqrt{6}} & \frac{1}{2 \sqrt{3}} & -\frac{1}{2 \sqrt{2}}
\end{array}\right)+\left(\epsilon_{13}^{L}\right)^{2}\left(\begin{array}{ccc}
-\frac{1}{\sqrt{6}} & -\frac{1}{2 \sqrt{3}} & 0 \\
0 & 0 & -\frac{1}{2 \sqrt{2}} \\
0 & 0 & -\frac{1}{2 \sqrt{2}}
\end{array}\right) \\
& +\epsilon_{12}^{L} \epsilon_{23}^{L}\left(\begin{array}{ccc}
0 & 0 & 0 \\
\frac{1}{\sqrt{3}} & \frac{1}{\sqrt{6}} & 0 \\
\frac{1}{\sqrt{3}} & \frac{1}{\sqrt{6}} & 0
\end{array}\right)+\epsilon_{12}^{L} \epsilon_{13}^{L} e^{i \delta^{L}}\left(\begin{array}{ccc}
0 & 0 & 0 \\
\frac{1}{\sqrt{6}} & -\frac{1}{\sqrt{3}} & 0 \\
\frac{1}{\sqrt{6}} & -\frac{1}{\sqrt{3}} & 0
\end{array}\right)+\epsilon_{23}^{L} \epsilon_{13}^{L} e^{i \delta^{L}}\left(\begin{array}{ccc}
0 & 0 & 0 \\
-\frac{1}{\sqrt{3}} & -\frac{1}{\sqrt{6}} & 0 \\
\frac{1}{\sqrt{3}} & \frac{1}{\sqrt{6}} & 0
\end{array}\right) \text {, }
\end{aligned}
$$


with $(-0.08)-0.04<\epsilon_{12}^{L}<0.01(-0.07), \epsilon_{23}^{L}, \epsilon_{13}^{L}, \delta^{L}$ are the same parameters to the parametrization of the PMNS matrix in Sec. III. This set of expansion parameters is certainly better than the one in the previous section if convergency is the criteria for the expansion.

With QLC, this leads to a very different $V_{0}$ for quark mixing than the Wolfenstein parametrization. In Ref. [20], we have derived the triminimal parametrization of the CKM matrix

$$
\begin{aligned}
& V_{\mathrm{CKM}}=V_{0}+\epsilon_{12}^{Q}\left(\begin{array}{ccc}
-\frac{\sqrt{2}-1}{\sqrt{6}} & \frac{\sqrt{2}+1}{\sqrt{6}} & 0 \\
-\frac{\sqrt{2}+1}{\sqrt{6}} & -\frac{\sqrt{2}-1}{\sqrt{6}} & 0 \\
0 & 0 & 0
\end{array}\right)+\epsilon_{23}^{Q}\left(\begin{array}{ccc}
0 & 0 & 0 \\
0 & 0 & 1 \\
\frac{\sqrt{2}-1}{\sqrt{6}} & -\frac{\sqrt{2}+1}{\sqrt{6}} & 0
\end{array}\right)+\epsilon_{13}^{Q}\left(\begin{array}{ccc}
0 & 0 & e^{-i \delta} \\
0 & 0 & 0 \\
-\frac{\sqrt{2}+1}{\sqrt{6}} e^{i \delta} & -\frac{\sqrt{2}-1}{\sqrt{6}} e^{i \delta} & 0
\end{array}\right) \\
& +\left(\epsilon_{12}^{Q}\right)^{2}\left(\begin{array}{ccc}
-\frac{\sqrt{2}+1}{2 \sqrt{6}} & -\frac{\sqrt{2}-1}{2 \sqrt{6}} & 0 \\
\frac{\sqrt{2}-1}{2 \sqrt{6}} & -\frac{\sqrt{2}+1}{2 \sqrt{6}} & 0 \\
0 & 0 & 0
\end{array}\right)+\left(\epsilon_{23}^{Q}\right)^{2}\left(\begin{array}{ccc}
0 & 0 & 0 \\
\frac{\sqrt{2}-1}{2 \sqrt{6}} & -\frac{\sqrt{2}+1}{2 \sqrt{6}} & 0 \\
0 & 0 & -\frac{1}{2}
\end{array}\right)+\left(\epsilon_{13}^{Q}\right)^{2}\left(\begin{array}{ccc}
-\frac{\sqrt{2}+1}{2 \sqrt{6}} & -\frac{\sqrt{2}-1}{2 \sqrt{6}} & 0 \\
0 & 0 & 0 \\
0 & 0 & -\frac{1}{2}
\end{array}\right) \\
& +\epsilon_{12}^{Q} \epsilon_{23}^{Q}\left(\begin{array}{ccc}
0 & 0 & 0 \\
0 & 0 & 0 \\
\frac{\sqrt{2}+1}{\sqrt{6}} & \frac{\sqrt{2}-1}{\sqrt{6}} & 0
\end{array}\right)+\epsilon_{12}^{Q} \epsilon_{13}^{Q} e^{i \delta}\left(\begin{array}{ccc}
0 & 0 & 0 \\
0 & 0 & 0 \\
\frac{\sqrt{2}-1}{\sqrt{6}} & -\frac{\sqrt{2}+1}{\sqrt{6}} & 0
\end{array}\right)+\epsilon_{23}^{Q} \epsilon_{13}^{Q} e^{i \delta}\left(\begin{array}{ccc}
0 & 0 & 0 \\
-\frac{\sqrt{2}+1}{\sqrt{6}} & -\frac{\sqrt{2}-1}{\sqrt{6}} & 0 \\
0 & 0 & 0
\end{array}\right) \text {, }
\end{aligned}
$$

with $\epsilon_{12}^{Q}=0.0577 \pm 0.0010, \epsilon_{23}^{Q}, \epsilon_{13}^{Q}, \delta^{Q}$ are the same parameters to the parametrization of the CKM matrix in Sec. III, and

$$
V_{0}=\left(\begin{array}{ccc}
\frac{\sqrt{2}+1}{\sqrt{6}} & \frac{\sqrt{2}-1}{\sqrt{6}} & 0 \\
-\frac{\sqrt{2}-1}{\sqrt{6}} & \frac{\sqrt{2}+1}{\sqrt{6}} & 0 \\
0 & 0 & 1
\end{array}\right)
$$

\section{B. The triminimal and Wolfenstein-like parametrizations}

In Ref. 15], the Wolfenstein-like parametrization of the PMNS matrix is also derived in two cases depending on the value of $\left|U_{e 3}\right|$. Let us discuss the relation between the two Wolfenstein-like parametrizations and the triminimal parametrization here.

If $\left|U_{e 3}\right|$ is of order $\lambda^{2}$, Case 1 in Ref. [15], after making the replacements of $\epsilon_{12}^{L}=-\sqrt{6}(\sqrt{2}-1) \lambda-3(\sqrt{2}-1)^{4} \lambda^{2}+$ $\mathcal{O}\left(\lambda^{3}\right), \epsilon_{23}^{L}=-A \lambda+\mathcal{O}\left(\lambda^{3}\right)$, and $\epsilon_{13}^{L} e^{i \delta^{L}}=A \lambda^{2}(\zeta+i \xi)+\mathcal{O}\left(\lambda^{3}\right)=A \lambda^{2} z+\mathcal{O}\left(\lambda^{3}\right)$, we have

$$
\epsilon_{13}^{L}=A \lambda^{2} \sqrt{\zeta^{2}+\xi^{2}}+\mathcal{O}\left(\lambda^{3}\right), \quad \epsilon_{12}^{L} \epsilon_{23}^{L}=\sqrt{6}(\sqrt{2}-1) A \lambda^{2}+\mathcal{O}\left(\lambda^{3}\right) .
$$

We obtain the parametrization of Case 1 of the PMNS matrix in Ref. [15] from Eq. (47)

$$
\begin{aligned}
U & =U_{\text {tri }}+\lambda\left(\begin{array}{ccc}
2-\sqrt{2} & -(2 \sqrt{2}-2) & 0 \\
2-\sqrt{2}-\frac{1}{\sqrt{6}} A & \sqrt{2}-1+\frac{1}{\sqrt{3}} A & -\frac{\sqrt{2}}{2} A \\
-(2-\sqrt{2})-\frac{1}{\sqrt{6}} A & -(\sqrt{2}-1)+\frac{1}{\sqrt{3}} A & \frac{\sqrt{2}}{2} A
\end{array}\right) \\
& +\lambda^{2}\left(\begin{array}{ccc}
-(15 \sqrt{6}-21 \sqrt{3}) & -(15 \sqrt{6}-21 \sqrt{3}) \\
15 \sqrt{3}-\frac{63}{\sqrt{6}}+\left(2-\sqrt{2}-\frac{z}{\sqrt{3}}\right) A+\frac{1}{2 \sqrt{6}} A^{2} & -\left(15 \sqrt{3}-\frac{63}{\sqrt{6}}\right)+\left(\sqrt{2}-1-\frac{z}{\sqrt{6}}\right) A-\frac{1}{2 \sqrt{3}} A^{2} & -\frac{\sqrt{2}}{4} A^{2} \\
-\left(15 \sqrt{3}-\frac{63}{\sqrt{6}}\right)+\left(2-\sqrt{2}-\frac{z}{\sqrt{3}}\right) A-\frac{1}{2 \sqrt{6}} A^{2} & 15 \sqrt{3}-\frac{63}{\sqrt{6}}+\left(\sqrt{2}-1-\frac{z}{\sqrt{6}}\right) A+\frac{1}{2 \sqrt{3}} A^{2} & -\frac{\sqrt{2}}{4} A^{2}
\end{array}\right) \\
& +\mathcal{O}\left(\lambda^{3}\right) .
\end{aligned}
$$

If $\left|U_{e 3}\right|$ is of order $\lambda$, Case 2 in Ref. [15], making the replacements of $\epsilon_{12}^{L}=-\sqrt{6}(\sqrt{2}-1) \lambda-3(\sqrt{2}-1)^{4} \lambda^{2}+\mathcal{O}\left(\lambda^{3}\right)$, $\epsilon_{23}^{L}=-A \lambda+\mathcal{O}\left(\lambda^{3}\right)$, and $\epsilon_{13}^{L} e^{i \delta^{L}}=A \lambda\left(\zeta^{\prime}+i \xi^{\prime}\right)+\mathcal{O}\left(\lambda^{3}\right)=A \lambda z^{\prime}+\mathcal{O}\left(\lambda^{3}\right)$, we have

$$
\epsilon_{13}^{L}=A \lambda \sqrt{\zeta^{\prime 2}+\xi^{\prime 2}}+\mathcal{O}\left(\lambda^{3}\right), \quad \epsilon_{12}^{L} \epsilon_{23}^{L}=\sqrt{6}(\sqrt{2}-1) A \lambda^{2}+\mathcal{O}\left(\lambda^{3}\right) .
$$

We obtain the parametrization of Case 2 of the PMNS matrix in Ref. [15] from Eq. (47)

$$
U=U_{\text {tri }}+\lambda\left(\begin{array}{ccc}
2-\sqrt{2} & -(2 \sqrt{2}-2) & z^{\prime *} A \\
2-\sqrt{2}-\left(\frac{1}{\sqrt{6}}+\frac{z^{\prime}}{\sqrt{3}}\right) A & \sqrt{2}-1+\left(\frac{1}{\sqrt{3}}-\frac{z^{\prime}}{\sqrt{6}}\right) A & -\frac{\sqrt{2}}{2} A \\
-(2-\sqrt{2})-\left(\frac{1}{\sqrt{6}}+\frac{z^{\prime}}{\sqrt{3}}\right) A & -(\sqrt{2}-1)+\left(\frac{1}{\sqrt{3}}-\frac{z^{\prime}}{\sqrt{6}}\right) A & \frac{\sqrt{2}}{2} A
\end{array}\right)
$$




$$
+\lambda^{2}\left(\begin{array}{ccc}
-(15 \sqrt{6}-21 \sqrt{3})-\frac{\sqrt{6}}{6}\left|z^{\prime}\right|^{2} A^{2} & -(15 \sqrt{6}-21 \sqrt{3})-\frac{\sqrt{3}}{6}\left|z^{\prime}\right|^{2} A^{2} & 0 \\
15 \sqrt{3}-\frac{63}{\sqrt{6}}+m A+p A^{2} & -\left(15 \sqrt{3}-\frac{63}{\sqrt{6}}\right)+n A-q A^{2} & -\frac{\sqrt{2}}{4}\left(1+\left|z^{\prime}\right|^{2}\right) A^{2} \\
-\left(15 \sqrt{3}-\frac{63}{\sqrt{6}}\right)+m A-p A^{2} & 15 \sqrt{3}-\frac{63}{\sqrt{6}}+n A+q A^{2} & -\frac{\sqrt{2}}{4}\left(1+\left|z^{\prime}\right|^{2}\right) A^{2}
\end{array}\right)+\mathcal{O}\left(\lambda^{3}\right)
$$

where

$$
m=2-\sqrt{2}-\frac{z^{\prime}}{\sqrt{2}+1}, \quad n=\sqrt{2}-1+\frac{2 z^{\prime}}{2+\sqrt{2}}, \quad p=\frac{1}{2 \sqrt{6}}+\frac{z^{\prime}}{\sqrt{3}}, \quad q=\frac{1}{2 \sqrt{3}}-\frac{z^{\prime}}{\sqrt{6}} .
$$

We have seen in both the last section and this section, the Wolfenstein-like parametrization of the PMNS matrix depends on how $U_{e 3}$ is parameterized. However, the triminimal parametrization is independent of how $U_{e 3}$ is parameterized making it easier to work with. One can obtain the Wolfenstein-like parametrization from the triminimal parametrization when appropriate definitions of the parameters are used.

For mixing in the quark sector, when taking the following definitions of the small parameters as, $\epsilon_{12}^{Q}=\sqrt{6}(\sqrt{2}-$ 1) $\lambda+3(\sqrt{2}-1)^{4} \lambda^{2}+\mathcal{O}\left(\lambda^{3}\right), \epsilon_{23}^{Q}=A \lambda+\mathcal{O}\left(\lambda^{3}\right)$, and $\epsilon_{13}^{Q} e^{i \delta^{L}}=A \lambda^{2}(\rho+i \eta)+\mathcal{O}\left(\lambda^{3}\right)$, we have

$$
\epsilon_{13}^{Q}=A \lambda^{2} \sqrt{\rho^{2}+\eta^{2}}+\mathcal{O}\left(\lambda^{3}\right), \quad \epsilon_{12}^{Q} \epsilon_{23}^{Q}=\sqrt{6}(\sqrt{2}-1) A \lambda^{2}+\mathcal{O}\left(\lambda^{3}\right) .
$$

Then we get the parametrization of the CKM matrix in Ref. [15]

$$
\begin{aligned}
V= & \left(\begin{array}{ccc}
\frac{\sqrt{2}+1}{\sqrt{6}} & \frac{\sqrt{2}-1}{\sqrt{6}} & 0 \\
-\frac{\sqrt{2}-1}{\sqrt{6}} & \frac{\sqrt{2}+1}{\sqrt{6}} & 0 \\
0 & 0 & 1
\end{array}\right)+\lambda\left(\begin{array}{ccc}
-(3-2 \sqrt{2}) & 1 & 0 \\
-1 & -(3-2 \sqrt{2}) & A \\
\frac{\sqrt{2}-1}{\sqrt{6}} A & -\frac{\sqrt{2}+1}{\sqrt{6}} A & 0
\end{array}\right) \\
& +\lambda^{2}\left(\begin{array}{ccc}
-(30 \sqrt{3}-21 \sqrt{6}) & 0 & (\rho-i \eta) A \\
\frac{\sqrt{2}-1}{2 \sqrt{6}} A^{2} & -(30 \sqrt{3}-21 \sqrt{6})-\frac{\sqrt{2}+1}{2 \sqrt{6}} A^{2} & 0 \\
\left(1-\frac{\sqrt{2}+1}{\sqrt{6}}(\rho+i \eta)\right) A & \left(3-2 \sqrt{2}-\frac{\sqrt{2}-1}{\sqrt{6}}(\rho+i \eta)\right) A & -\frac{1}{2} A^{2}
\end{array}\right)+\mathcal{O}\left(\lambda^{3}\right) .
\end{aligned}
$$

The Jarlskog parameters for quark and lepton have been obtained in Ref. [20] and Ref. [16] (to lowest order of $\left.\epsilon_{i j}^{Q, L}\right)$, respectively.

$$
\begin{aligned}
J^{Q} & =\left(\frac{1}{6}+\frac{2 \sqrt{2}}{3} \epsilon_{12}^{Q}\right) \epsilon_{23}^{Q} \epsilon_{13}^{Q} \sin \delta^{Q} . \\
J^{L} & =\left(\frac{1}{3 \sqrt{2}}+\frac{1}{6} \epsilon_{12}^{L}\right) \epsilon_{13}^{L} \sin \delta^{L} .
\end{aligned}
$$

\section{DISCUSSIONS AND CONCLUSIONS}

In the literature there are several different zeroth order basis for the CKM and PMNS matrices. Each of them has its virtual. We would like to comment on a few of them before drawing our conclusions. We find that although some of these parametrizations converge faster than the ones in the previous two sections, they are in general very complicated in expression for perturbation series and difficult to use.

The first case is a variant of QLC. Petcov and Smirnov [21] noticed that $\tan 2 \theta_{12}^{L}=1 / \tan 2 \theta_{12}^{Q}$. This is in fact another form of QLC. In Ref. [22], specific values for the zeroth order angles are given with

$$
\tan 2 \theta_{12}^{Q}=\frac{1}{2}, \quad \tan 2 \theta_{12}^{L}=2 .
$$

which gives that $\sin \theta_{12}^{Q}=0.2298$ and agrees very well with $\sin \theta_{12}^{Q}=0.2257 \pm 0.0010$ obtained from Eq. (5). In this case $\epsilon_{12}^{Q}$ is smaller than the cases discussed in Sections III and IV. From convergency point of view this may be a better zeroth order basis. We have worked out the corresponding basis $V_{0}$ in Appendix A. It can be seen that the zeroth order basis is complicated which makes one wonder if it is a good way to expand the mixing matrix. The expansion is too complicated to be useful as can be seen from the results displayed in Appendix A. 
There are also parameterizations for quark and lepton mixing without reference to QLC. We comment on two cases here. One of them is to set [23]

$$
\begin{array}{lll}
\tan 2 \theta_{12}^{Q}=\frac{\sqrt{2}}{3}, & \theta_{23}^{Q}=0, & \theta_{13}^{Q}=0, \\
\tan \theta_{12}^{L}=\frac{\sqrt{2}}{2}, & \theta_{23}^{L}=\frac{\pi}{4}, & \theta_{13}^{L}=0 .
\end{array}
$$

The above set of parametrization violate the QLC relations since $\theta_{12}^{Q}+\theta_{12}^{L}=47.9^{\circ}$. The basis matrix for the PMNS matrix is still the tri-bimaximal mixing matrix, and the triminimal parametrization of the PMNS matrix has already been derived in Ref. [16]. However, as can be seen from Appendix B, the basis matrix for quark mixing is very complicated. One concludes again that this way of expanding the mixing matrix is too complicated to be practical for using. The above two basis can not be considered, at least, as convenient ones to use.

Another is the case discussed in Ref. [24] where the basis angles are chosen to be

$$
\begin{array}{lll}
\theta_{12}^{Q}=\frac{\pi}{12}, & \theta_{23}^{Q}=0, & \theta_{13}^{Q}=0 \\
\theta_{12}^{L}=\frac{\pi}{5}, & \theta_{23}^{L}=\frac{\pi}{4}, & \theta_{13}^{L}=0
\end{array}
$$

It is interesting to note that the angle $\pi / 5$ gives $\cos \left(\theta_{12}^{L}\right)$ to be half of the golden ratio $(1+\sqrt{5}) / 2$. The basis matrices are reasonably simple.

The above two cases, however, leave the basis matrices for quarks and leptons unrelated. If one is looking for a unified parametrization for quark and lepton sectors, this may not be a good way to go.

We now summarize the main results. We have derived the general expressions of the triminimal parametrizations of the mixing matrix for quark and lepton sectors. The triminimal parametrization is an approximate expansion based on the standard parametrization of the mixing matrix in three angle parameters and one phase parameter. When the zeroth order basis of the mixing matrix is determined, the parameters of the triminimal parametrization are fixed.

Compared with the familiar Wolfenstein-like parametrization, the triminimal parametrization has the advantages of uniqueness and simplicity. In Wolfenstein (-like) parametrizations, the correction terms are put in by hand with certain physical consideration taken into account. The explicit form has ambiguities depending on one's preference of physical considerations even the zeroth order basis matrices are chosen. This makes the use of the parametrization more complicated. Of course if the parametrizations have been done consistently, different parametrizations must be able to transfer from one to another. We have explicitly shown how to identify the connections of parameters in the triminimal and Wolfenstein (-like) parametrizations for several interesting choices of basis matrices, the unit, bimaximal and tri-bimaximal cases. The Wolfenstein-like parametrizations derived from triminimal parametrizations have the advantage of the uniqueness whereas as convenient to use as traditional Wolfenstein parametrization in practical applications.

A priori, parametrization for quark and lepton sectors are seemly not connected. However, if connection can be made, it may provide a hint for the underlying theory generating mixing. The QLC relations provide a useful guide. We find that the unit basis for quark mixing corresponds to the bimaximal basis for lepton mixing. Present experimental data indicate that the tri-bimaximal pattern represents mixing in the lepton sector very well. We have therefore studied the corresponding basis matrix in the quark sector and compared with previous studies based on Wolfenstein-like parametrizations. If one imposes the condition that the parametrization must satisfy the QLC relations even when corrections to the basis matrices are included, then the corrections $\epsilon_{i j}^{Q}$ for quark and $\epsilon_{i j}^{L}$ for lepton sectors are related by $\epsilon_{12}^{Q}+\epsilon_{12}^{L}=0$ and $\epsilon_{23}^{Q}+\epsilon_{23}^{L}=0$. One should keep in mind that the corrections are not necessarily required to satisfy these relations. With more accurate data to be available in the near future, the QLC relations can be tested.

For perturbative expansions of the CKM and PMNS matrices, one should use simple zeroth order basis matrices and the expansions should have a fast convergence. In the literature, many other zeroth order basis matrices have been proposed. We find some of them very complicated to use and some of them have the quark and lepton mixing matrix unrelated. We find that the pair simple zeroth order basis matrices, the unit matrix for quarks and bimaximal matrix for leptons, are convenient to use with reasonable convergency. The pair simple zeroth order basis matrices, the tri-bimaximal matrix for leptons and its QLC partner for quarks, make the expansions converge faster. We consider these two sets of zeroth basis matrices as good starting point for perturbative parametrizations for the CKM and PMNS matrices. It would be interesting to see if theoretical progresses can realize such connections. 


\section{Acknowledgments}

This work was partially supported by NSFC (Nos. 10721063, 10575003, 10528510), by the Key Grant Project of Chinese Ministry of Education (No. 305001), by NSC, and by NCTS.

\section{APPENDIX A}

The basis matrices for the CKM and PMNS matrices with $\tan 2 \theta_{12}^{Q}=\frac{1}{2}$ and $\tan 2 \theta_{12}^{L}=2$ are

$$
\begin{aligned}
V_{\mathrm{CKMb}} & =\left(\begin{array}{ccc}
(10-4 \sqrt{5})^{-1 / 2} & \frac{1}{2 \sqrt{5}}(10-4 \sqrt{5})^{1 / 2} & 0 \\
-\frac{1}{2 \sqrt{5}}(10-4 \sqrt{5})^{1 / 2} & (10-4 \sqrt{5})^{-1 / 2} & 0 \\
0 & 0 & 1
\end{array}\right), \\
U_{\mathrm{PMNSb}} & =\left(\begin{array}{ccc}
2(10-2 \sqrt{5})^{-1 / 2} & \frac{1}{2 \sqrt{5}}(10-2 \sqrt{5})^{1 / 2} & 0 \\
-\frac{1}{2 \sqrt{10}}(10-4 \sqrt{5})^{1 / 2} & \sqrt{2}(10-2 \sqrt{5})^{-1 / 2} & \frac{\sqrt{2}}{2} \\
\frac{1}{2 \sqrt{10}}(10-4 \sqrt{5})^{1 / 2} & -\sqrt{2}(10-2 \sqrt{5})^{-1 / 2} & \frac{\sqrt{2}}{2}
\end{array}\right) .
\end{aligned}
$$

We see that the basis matrices are very complicated.

For triminimal expansion, we set

$$
\begin{aligned}
& \theta_{12}^{Q}=\arctan (\sqrt{5}-2)+\epsilon_{12}^{Q}, \quad \theta_{23}^{Q}=\epsilon_{23}^{Q}, \quad \theta_{13}^{Q}=\epsilon_{13}^{Q}, \\
& \theta_{12}^{L}=\arctan \frac{\sqrt{5}-1}{2}+\epsilon_{12}^{L}, \quad \theta_{23}^{L}=\frac{\pi}{4}+\epsilon_{23}^{L}, \quad \theta_{13}^{L}=\epsilon_{13}^{L},
\end{aligned}
$$

then we have

$$
\begin{aligned}
V_{\mathrm{CKM}} & =R_{23}\left(\epsilon_{23}^{Q}\right) U_{\delta}^{\dagger} R_{13}\left(\epsilon_{13}^{Q}\right) U_{\delta} R_{12}\left(\epsilon_{12}^{Q}\right) R_{12}(\arctan (\sqrt{5}-2)) \\
U_{\mathrm{PMNS}} & =R_{23}\left(\frac{\pi}{4}\right) R_{23}\left(\epsilon_{23}^{L}\right) U_{\delta}^{\dagger} R_{13}\left(\epsilon_{13}^{L}\right) U_{\delta} R_{12}\left(\epsilon_{12}^{L}\right) R_{12}\left(\arctan \frac{\sqrt{5}-1}{2}\right) .
\end{aligned}
$$

To second order in $\epsilon_{i j}$, we have

$$
\begin{aligned}
& V_{\mathrm{CKM}}=V_{\mathrm{CKMb}}+\epsilon_{12}^{Q}\left(\begin{array}{ccc}
-\frac{1}{2 \sqrt{5}}(10-4 \sqrt{5})^{1 / 2} & (10-4 \sqrt{5})^{-1 / 2} & 0 \\
-(10-4 \sqrt{5})^{-1 / 2} & -\frac{1}{2 \sqrt{5}}(10-4 \sqrt{5})^{1 / 2} & 0 \\
0 & 0 & 0
\end{array}\right) \\
& +\epsilon_{23}^{Q}\left(\begin{array}{ccc}
0 & 0 \\
\frac{1}{2 \sqrt{5}}(10-4 \sqrt{5})^{1 / 2} & -(10-4 \sqrt{5})^{-1 / 2} & 0
\end{array}\right)+\epsilon_{13}^{Q}\left(\begin{array}{ccc}
0 & 0 & e^{-i \delta^{Q}} \\
0 & 0 & 0 \\
-(10-4 \sqrt{5})^{-1 / 2} e^{i \delta^{Q}} & -\frac{1}{2 \sqrt{5}}(10-4 \sqrt{5})^{1 / 2} e^{i \delta^{Q}} & 0
\end{array}\right) \\
& +\left(\epsilon_{12}^{Q}\right)^{2}\left(\begin{array}{ccc}
-\frac{1}{2}(10-4 \sqrt{5})^{-1 / 2} & -\frac{1}{4 \sqrt{5}}(10-4 \sqrt{5})^{1 / 2} & 0 \\
\frac{1}{4 \sqrt{5}}(10-4 \sqrt{5})^{1 / 2} & -\frac{1}{2}(10-4 \sqrt{5})^{-1 / 2} & 0 \\
0 & 0 & 0
\end{array}\right)+\left(\epsilon_{23}^{Q}\right)^{2}\left(\begin{array}{ccc}
0 & 0 \\
\frac{1}{4 \sqrt{5}}(10-4 \sqrt{5})^{1 / 2} & -\frac{1}{2}(10-4 \sqrt{5})^{-1 / 2} & 0 \\
0 & 0 & -\frac{1}{2}
\end{array}\right) \\
& +\left(\epsilon_{13}^{Q}\right)^{2}\left(\begin{array}{ccc}
-\frac{1}{2}(10-4 \sqrt{5})^{-1 / 2} & -\frac{1}{4 \sqrt{5}}(10-4 \sqrt{5})^{1 / 2} & 0 \\
0 & 0 & 0 \\
0 & 0 & -\frac{1}{2}
\end{array}\right)+\epsilon_{12}^{Q} \epsilon_{23}^{Q}\left(\begin{array}{cccc}
0 & 0 & 0 \\
0 & 0 & 0 \\
(10-4 \sqrt{5})^{-1 / 2} & \frac{1}{2 \sqrt{5}}(10-4 \sqrt{5})^{1 / 2} & 0
\end{array}\right) \\
& +\epsilon_{12}^{Q} \epsilon_{13}^{Q} e^{i \delta^{Q}}\left(\begin{array}{ccc}
0 & 0 \\
\frac{1}{2 \sqrt{5}}(10-4 \sqrt{5})^{1 / 2} & -(10-4 \sqrt{5})^{-1 / 2} & 0
\end{array}\right)+\epsilon_{23}^{Q} \epsilon_{13}^{Q} e^{i \delta^{Q}}\left(\begin{array}{ccc}
0 & 0 \\
-(10-4 \sqrt{5})^{-1 / 2} & -\frac{1}{2 \sqrt{5}}(10-4 \sqrt{5})^{1 / 2} & 0 \\
0 & 0
\end{array}\right) \\
& +\mathcal{O}\left(\left(\epsilon_{i j}^{Q}\right)^{3}\right) \text {. } \\
& U_{\mathrm{PMNS}}=U_{\mathrm{PMNSb}}+\epsilon_{12}^{L}\left(\begin{array}{ccc}
-\frac{1}{2 \sqrt{5}}(10-2 \sqrt{5})^{1 / 2} & 2(10-2 \sqrt{5})^{-1 / 2} & 0 \\
-\sqrt{2}(10-2 \sqrt{5})^{-1 / 2} & -\frac{1}{2 \sqrt{10}}(10-2 \sqrt{5})^{1 / 2} & 0 \\
\sqrt{2}(10-2 \sqrt{5})^{-1 / 2} & \frac{1}{2 \sqrt{10}}(10-2 \sqrt{5})^{1 / 2} & 0
\end{array}\right)
\end{aligned}
$$




$$
\begin{aligned}
& +\epsilon_{23}^{L}\left(\begin{array}{ccc}
0 & 0 & 0 \\
\frac{1}{2 \sqrt{10}}(10-2 \sqrt{5})^{1 / 2} & -\sqrt{2}(10-2 \sqrt{5})^{-1 / 2} & \frac{\sqrt{2}}{2} \\
\frac{1}{2 \sqrt{10}}(10-2 \sqrt{5})^{1 / 2} & -\sqrt{2}(10-2 \sqrt{5})^{-1 / 2} & -\frac{\sqrt{2}}{2}
\end{array}\right) \\
& +\epsilon_{13}^{L}\left(\begin{array}{ccc}
0 & 0 & e^{-i \delta^{L}} \\
-\sqrt{2}(10-2 \sqrt{5})^{-1 / 2} e^{i \delta^{L}} & -\frac{1}{2 \sqrt{10}}(10-2 \sqrt{5})^{1 / 2} e^{i \delta^{L}} & 0 \\
-\sqrt{2}(10-2 \sqrt{5})^{-1 / 2} e^{i \delta^{L}} & -\frac{1}{2 \sqrt{10}}(10-2 \sqrt{5})^{1 / 2} e^{i \delta^{L}} & 0
\end{array}\right) \\
& +\left(\epsilon_{12}^{L}\right)^{2}\left(\begin{array}{ccc}
-(10-2 \sqrt{5})^{-1 / 2} & -\frac{1}{4 \sqrt{5}}(10-2 \sqrt{5})^{1 / 2} & 0 \\
\frac{1}{4 \sqrt{10}}(10-2 \sqrt{5})^{1 / 2} & -\frac{1}{\sqrt{2}}(10-2 \sqrt{5})^{-1 / 2} & 0 \\
-\frac{1}{4 \sqrt{10}}(10-2 \sqrt{5})^{1 / 2} & \frac{1}{\sqrt{2}}(10-2 \sqrt{5})^{-1 / 2} & 0
\end{array}\right)+\left(\epsilon_{23}^{L}\right)^{2}\left(\begin{array}{ccc}
0 & 0 \\
\frac{1}{4 \sqrt{10}}(10-2 \sqrt{5})^{1 / 2} & -\frac{1}{\sqrt{2}}(10-2 \sqrt{5})^{-1 / 2} & -\frac{\sqrt{2}}{4} \\
-\frac{1}{4 \sqrt{10}}(10-2 \sqrt{5})^{1 / 2} & \frac{1}{\sqrt{2}}(10-2 \sqrt{5})^{-1 / 2} & -\frac{\sqrt{2}}{4}
\end{array}\right) \\
& +\left(\epsilon_{13}^{L}\right)^{2}\left(\begin{array}{ccc}
-(10-2 \sqrt{5})^{-1 / 2} & -\frac{1}{4 \sqrt{5}}(10-2 \sqrt{5})^{1 / 2} & 0 \\
0 & 0 & -\frac{\sqrt{2}}{4} \\
0 & 0 & -\frac{\sqrt{2}}{4}
\end{array}\right)+\epsilon_{12}^{L} \epsilon_{23}^{L}\left(\begin{array}{ccc}
0 & 0 & 0 \\
\sqrt{2}(10-2 \sqrt{5})^{-1 / 2} & \frac{1}{2 \sqrt{10}}(10-2 \sqrt{5})^{1 / 2} & 0 \\
\sqrt{2}(10-2 \sqrt{5})^{-1 / 2} & \frac{1}{2 \sqrt{10}}(10-2 \sqrt{5})^{1 / 2} & 0
\end{array}\right) \\
& +\epsilon_{12}^{L} \epsilon_{13}^{L} e^{i \delta^{L}}\left(\begin{array}{ccc}
0 & 0 & 0 \\
\frac{1}{2 \sqrt{10}}(10-2 \sqrt{5})^{1 / 2} & -\sqrt{2}(10-2 \sqrt{5})^{-1 / 2} & 0 \\
\frac{1}{2 \sqrt{10}}(10-2 \sqrt{5})^{1 / 2} & -\sqrt{2}(10-2 \sqrt{5})^{-1 / 2} & 0
\end{array}\right) \\
& +\epsilon_{23}^{L} \epsilon_{13}^{L} e^{i \delta^{L}}\left(\begin{array}{ccc}
0 & 0 & 0 \\
-\sqrt{2}(10-2 \sqrt{5})^{-1 / 2} & -\frac{1}{2 \sqrt{10}}(10-2 \sqrt{5})^{1 / 2} & 0 \\
\sqrt{2}(10-2 \sqrt{5})^{-1 / 2} & \frac{1}{2 \sqrt{10}}(10-2 \sqrt{5})^{1 / 2} & 0
\end{array}\right)+\mathcal{O}\left(\left(\epsilon_{i j}^{L}\right)^{3}\right) .
\end{aligned}
$$

\section{APPENDIX B}

With the following angles for the zeroth order expansion

$$
\begin{aligned}
& \tan 2 \theta_{12}^{Q}=\frac{\sqrt{2}}{3}, \quad \theta_{23}^{Q}=0, \quad \theta_{13}^{Q}=0, \\
& \tan \theta_{12}^{L}=\frac{\sqrt{2}}{2}, \quad \theta_{23}^{L}=\frac{\pi}{4}, \quad \theta_{13}^{L}=0 .
\end{aligned}
$$

we obtain the basis matrix for the CKM matrix

$$
V_{\mathrm{CKMb}}=\left(\begin{array}{ccc}
\frac{1}{11^{1 / 4}}(\sqrt{11}-3)^{-1 / 2} & \frac{1}{11^{1 / 4} \sqrt{2}}(\sqrt{11}-3)^{1 / 2} & 0 \\
-\frac{1}{11^{1 / 4} \sqrt{2}}(\sqrt{11}-3)^{1 / 2} & \frac{1}{11^{1 / 4}}(\sqrt{11}-3)^{-1 / 2} & 0 \\
0 & 0 & 1
\end{array}\right)
$$

and

$$
V_{\mathrm{CKM}}=R_{23}\left(\epsilon_{23}^{Q}\right) U_{\delta}^{\dagger} R_{13}\left(\epsilon_{13}^{Q}\right) U_{\delta} R_{12}\left(\epsilon_{12}^{Q}\right) R_{12}\left(\arctan \frac{\sqrt{11}-3}{\sqrt{2}}\right) .
$$

The triminimal parametrization of the CKM matrix is

$V_{\mathrm{CKM}}$

$=V_{\mathrm{CKMb}}+\frac{\epsilon_{12}^{Q}}{11^{1 / 4}}\left(\begin{array}{ccc}-\frac{1}{\sqrt{2}}(\sqrt{11}-3)^{1 / 2} & (\sqrt{11}-3)^{-1 / 2} & 0 \\ -(\sqrt{11}-3)^{-1 / 2} & -\frac{1}{\sqrt{2}}(\sqrt{11}-3)^{1 / 2} & 0 \\ 0 & 0 & 0\end{array}\right)+\frac{\epsilon_{23}^{Q}}{11^{1 / 4}}\left(\begin{array}{ccc}0 & 0 & 0 \\ 0 & 1 \\ \frac{1}{\sqrt{2}}(\sqrt{11}-3)^{1 / 2} & -(\sqrt{11}-3)^{-1 / 2} & 0\end{array}\right)$ $+\frac{\epsilon_{13}^{Q}}{11^{1 / 4}}\left(\begin{array}{ccc}0 & 0 & 11^{1 / 4} e^{-i \delta^{Q}} \\ 0 & 0 & 0 \\ -(\sqrt{11}-3)^{-1 / 2} e^{i \delta^{Q}} & -\frac{1}{\sqrt{2}}(\sqrt{11}-3)^{1 / 2} e^{i \delta^{Q}} & 0\end{array}\right)$

$+\frac{\left(\epsilon_{12}^{Q}\right)^{2}}{11^{1 / 4}}\left(\begin{array}{ccc}-\frac{1}{2}(\sqrt{11}-3)^{-1 / 2} & -\frac{1}{2 \sqrt{2}}(\sqrt{11}-3)^{1 / 2} & 0 \\ \frac{1}{2 \sqrt{2}}(\sqrt{11}-3)^{1 / 2} & -\frac{1}{2}(\sqrt{11}-3)^{-1 / 2} & 0 \\ 0 & 0 & 0\end{array}\right)+\frac{\left(\epsilon_{23}^{Q}\right)^{2}}{11^{1 / 4}}\left(\begin{array}{ccc}0 & 0 \\ \frac{1}{2 \sqrt{2}}(\sqrt{11}-3)^{1 / 2} & -\frac{1}{2}(\sqrt{11}-3)^{-1 / 2} & 0 \\ 0 & 0 & -\frac{11^{1 / 4}}{2}\end{array}\right)$ 


$$
\begin{aligned}
& +\frac{\left(\epsilon_{13}^{Q}\right)^{2}}{11^{1 / 4}}\left(\begin{array}{ccc}
-\frac{1}{2}(\sqrt{11}-3)^{-1 / 2} & -\frac{1}{2 \sqrt{2}}(\sqrt{11}-3)^{1 / 2} & 0 \\
0 & 0 & 0 \\
0 & 0 & -\frac{11^{1 / 4}}{2}
\end{array}\right)+\frac{\epsilon_{12}^{Q} \epsilon_{23}^{Q}}{11^{1 / 4}}\left(\begin{array}{ccc}
0 & 0 & 0 \\
0 & 0 & 0 \\
(\sqrt{11}-3)^{-1 / 2} & \frac{1}{\sqrt{2}}(\sqrt{11}-3)^{1 / 2} & 0
\end{array}\right) \\
& +\frac{\epsilon_{12}^{Q} \epsilon_{13}^{Q} e^{i \delta^{Q}}}{11^{1 / 4}}\left(\begin{array}{ccc}
0 & 0 \\
0 & 0 & 0 \\
\frac{1}{\sqrt{2}}(\sqrt{11}-3)^{1 / 2} & -(\sqrt{11}-3)^{-1 / 2} & 0
\end{array}\right)+\frac{\epsilon_{23}^{Q} \epsilon_{13}^{Q} e^{i \delta^{Q}}}{11^{1 / 4}}\left(\begin{array}{ccc}
0 \\
-(\sqrt{11}-3)^{-1 / 2} & -\frac{1}{\sqrt{2}}(\sqrt{11}-3)^{1 / 2} & 0 \\
0 & 0 & 0
\end{array}\right) \\
& +\mathcal{O}\left(\left(\epsilon_{i j}^{Q}\right)^{3}\right) .
\end{aligned}
$$

[1] N. Cabibbo, Phys. Rev. Lett. 10, 531 (1963).

[2] M. Kobayashi and T. Maskawa, Prog. Theor. Phys. 49, 652 (1973).

[3] B. Pontecorvo, Sov. Phys. JETP 6, 429 (1957).

[4] Z. Maki, M. Nakagawa, and S. Sakata, Prog. Theor. Phys. 28, 870 (1962).

[5] L.L. Chau and W.Y. Keung, Phys. Rev. Lett. 53, 1802 (1984).

[6] Particle Data Group, C. Amsler et al., Phys. Lett. B667, 1 (2008).

[7] M. Bonna et al., http://www.utfit.org.

[8] M.C. Gonzalez-Garcia and M. Maltoni, Phys. Rept. 460, 1 (2008).

[9] L. Wolfenstein, Phys. Rev. Lett. 51, 1945 (1983).

[10] F. Vissani, hep-ph/9708483; V.D. Barger, S.Pakvasa, T.J. Weiler, and K. Whisnant, Phys. Lett. B437, 107 (1998); A.J. Baltz, A.S. Goldhaber, and M. Goldhaber, Phys. Rev. Lett. 81, 5730 (1998); I. Stancu and D.V. Ahluwalia, Phys. Lett. B460, 431 (1999); H. Georgi and S.L. Glashow, Phy. Rev. D61, 097301 (2000).

[11] P.F. Harrison, D.H. Perkins, and W.G. Scott, Phys. Lett. B458, 79 (1999); Phys. Lett. B530, 167 (2002); Z.Z. Xing, Phys. Lett. B533, 85 (2002); P.F. Harrison and W.G. Scott, Phys. Lett. B535, 163 (2002); Phys. Lett. B557, 76 (2003); X.-G. He and A. Zee, Phys. Lett. B560, 87 (2003). See also L. Wolfenstein, Phys. Rev. D18, 958 (1978); Y. Yamanaka, H. Sugawara, and S. Pakvasa, Phys. Rev. D25, 1895(1982); D29, 2135(E)(1984).

[12] W. Rodejohann, Phys. Rev. D69, 033005 (2004); N. Li and B.-Q. Ma, Phys. Lett. B600, 248 (2004).

[13] N. Li and B.-Q. Ma, Phys. Rev. D71, 097301 (2005).

[14] N. Li and B.-Q. Ma, Phys. Rev. D71, 017302 (2005).

[15] S.-W. Li and B.-Q. Ma, Phys. Rev. D77, 093005 (2008).

[16] S. Pakvasa, W. Rodejohann, and T.J. Weiler, Phy. Rev. Lett. 100, 111801 (2008). An equivalent triminimal parametrization was also give in, S.F. King, Phys. Lett. B659, 244 (2008).

[17] A.Y. Smirnov, hep-ph/0402264.

[18] H. Minakata and A.Y. Smirnov, Phys. Rev. D70, 073009 (2004); M. Raidal, Phys. Rev. Lett. 93, 161801 (2004); P.H. Frampton and R.N. Mohapatra, JHEP 0501, 025 (2005); J. Ferrandis and S. Pakvasa, Phys. Rev. D71, 033004 (2005); S.K. Kang, C.S. Kim and J. Lee, Phys. Lett. B619, 129 (2005); S. Antusch, S.F. King and R.N. Mohapatra, Phys. Lett. B618, 150 (2005); M.A. Schmidt and A.Y. Smirnov, Phys. Rev. D74, 113003 (2006); F. Plentinger, G. Seidl and W. Winter, Phys. Rev. D76, 113003 (2007).

[19] C. Jarlskog, Phys. Rev. Lett. 55, 1039 (1985); Z. Phys. C29, 491 (1985).

[20] X.-G. He, S.-W. Li and B.-Q. Ma, Phys. Rev. D78, 111301(R) (2008).

21] S.T. Petcov and A.Yu. Smirnov, Phys. Lett. B322, 109 (1994).

[22] Q. Duret, B. Machet, and M.I. Vysotsky, arXiv:0805.4121 [hep-ph].

[23] P.H. Frampton, T.W. Kephart, S. Matsuzaki, Phys. Rev. D78, 073004 (2008).

[24] W. Rodejohann, Phys. Lett. B671, 267 (2009). 